\title{
Supervivencia de empresas sociales de nueva creación. Un enfoque basado en el análisis cualitativo comparativo fsQCA
}

\author{
Rafael Fernández Guerrero \\ Lorenzo Revuelto Taboada \\ Virginia Simón Moya
}

RESUMEN: Las empresas sociales constituyen una realidad de la que multitud de instituciones, tanto organismos internacionales como universidades de prestigio, se han hecho eco dedicando recursos a su estudio y promoción. Actualmente, la literatura se centra, fundamentalmente, en investigar las diferencias entre emprendimiento de negocios y social, las diferentes lógicas de las empresas que, siendo sociales, persiguen fines lucrativos con el fin de obtener un sistema sostenible, o la medición del impacto del valor social que aportan. Sin embargo, poco se ha investigado aún sobre cómo afectan determinadas características del emprendedor y la empresa social a supervivencia, lo que constituye el fin de este trabajo. El estudio utiliza una muestra que oscila entre 291 y 212 empresas según el periodo considerado. Utilizando el Análisis Cualitativo Comparativo, tratamos de identificar si existe algún antecedente que sea condición necesaria para la supervivencia, así como combinaciones de antecedentes causales capaces de explicar la supervivencia. Los resultados muestran que no existe ninguna condición necesaria por sí misma y que existen diversas soluciones suficientes que varían conforme la empresa adquiere mayor madurez.

PALABRAS CLAVE: Emprendimiento social, supervivencia empresarial, características del emprendedor, tamaño, plan de empresa, fsQCA.

CLAVES ECONLIT: M13, L31, L25.

Cómo citar este artículo / How to cite this article: FERNÁNDEZ-GUERRERO, R., REVUELTO-TABOADA, L. \&

SIMÓN-MOYA, V. (2018): "Supervivencia de empresas sociales de nueva creación. Un enfoque basado en el análisis cualitativo comparativo fsQCA", CIRIEC-España, Revista de Economía Pública, Social y Cooperativa, 92, 183-221.

Correspondencia: Rafael Fernández Guerrero, Doctor en Ciencias Económicas y Empresariales, Universitat de València (España); Lorenzo Revuelto Taboada, Doctor en Ciencias Económicas y Empresariales, Universitat de València (España), lorenzo.revuelto@uv.es, y Virginia Simón Moya, Doctora en Dirección de Empresas, Universitat de València (España). 


\section{EXPANDED ABSTRACT}

\section{Survival of new social ventures. An approach based on qualitative comparative analysis fsQCA}

\section{Objectives}

This article has as main objective to check how some entrepreneur's and venture characteristics are related to new social venture survival. Specifically, related to the venture, five conditions have been studied, these conditions are: venture workforce, initial capital and three variables that assess the quality of the social venture plan (business plan). Regarding the entrepreneur's conditions, the experience related to the new venture activity, training level and specific training, and entrepreneurship motivation have been taken into account.

\section{Study design and methodology}

The study uses a sample that oscillates between 291 and 212 start-ups according to the period considered. The two outcome original variables are survival at 31 December of the third year $(\mathrm{ST}+3)$ and at 31 December of the sixth year $(\mathrm{ST}+6)$ after the new venture foundation. Both variables are dichotomous ( 1 = survival; 0 = failure).

The access to this sample has been possible with the collaboration of the Program Management and Planning Service from the Valencia Institute of Youth (IVAJ). This institution provided data on these ventures, whose creators were young entrepreneurs under 30 years old, or under 30 years but partnered with co-workers aged over 30 years, who applied for a grant.

Our propositions or tenets imply the existence of an interplay between business plan quality and entrepreneur and firm characteristics. In this case, different combinations of causal conditions could lead to the same outcome. Therefore, taking into account the limitations of conventional quantitative methods to study the effect of different possible causal recipes on survival, this study uses Qualitative Comparative Analysis, specifically fsQCA. QCA methods build and test theories of complex antecedent conditions that are sufficient or necessary to explain an outcome condition (e.g., firm survival). That is, the goal is to determine if any of the conditions, or a combination of the conditions that have been numbered in the previous section are sufficient or necessary to explain new social ventures' survival. 


\section{Results}

Due to the nature of the analysis that has been applied, the results can be divided into two blocks. The first one come from testing whether any of the causal conditions and their negation are necessary for the 3-year and 6-year survival. Our results show that any of the antecedent conditions is necessary or "almost necessary" for any outcome condition because any consistency score exceeds the threshold of 0.9 .

The second group of results shows models of sufficiency analysis. In ST+3 there are four main causal configurations that lead to firm survival. There are some conditions that are present in the four combinations, these conditions are both specific training and the level of entrepreneur's training, economic and organizational viability and initial capital. On the other hand, one of the combinations shows a curious characteristic, that is, the lack of financial viability makes the likelihood of survival grow. The most logic explanation is that as we are analyzing social ventures, and that these organizations trust more on subventions and public helps that on self-financing.

At $t+6$, as at $t+3$ there are also 4 causal configurations that lead to firm survival. From the results it can be extracted that, specific or related training and training level, and economic and organizational viability are important conditions in explaining new venture survival. These results are similar to those obtained in the short run. However, there are one of the conditions that loose importance in the medium run, initial capital. Contrarily, related experience, which was not a key antecedent condition at ST+3 appears in the four relevant causal combinations in ST+6.

\section{Limitations of the study}

The limitations of the study are mainly related to the sample. The first limitation comes from the nature of the entrepreneurs; the sample is composed by entrepreneurs who live in the Valencian Community and who are all under 30 , or under 30 but partnered with co-workers aged over 30 years. That characteristic of the sample makes difficult the extrapolation of the results at other territories and types of entrepreneurs. It is therefore advisable for subsequent research to replicate the study in a different geographical area, employing a sample comprising entrepreneurs of any age, which would probably make previous experience play a more determinant role when explaining new social venture survival. Likewise, it would be advisable to examine the real role played by the business plan, that is, if new ventures use it for something more than as a means of obtaining public support.

\section{Conclusions}

The most important conclusion is that any of the studied antecedent conditions alone is necessary or almost necessary, but these conditions take part of diverse overlapping causal configuration sufficient for displaying survival of both new and established social ventures. In fact, 
there are some antecedent conditions that are relevant in the short and medium run. These conditions are related to the entrepreneur's background, his/her training and experience. Moreover, the economic and organizational viability of the social venture plan are also important. One of the most important conditions at the short run, initial capital, loses importance at the long run. Probably because there are new social ventures that survive in the short run because of their amount of financial capital, but are not able to generate resources to be reinvested in a longer run.

fsQCA offers a deeper understanding of the role of the different antecedent conditions, because it allows analyzing the effects of combining indicators of the quality of the business plan with entrepreneur and business characteristics. Furthermore, this technique allows researchers to consider different combinations of initial conditions that are able to foster firm survival probabilities, a result impossible to obtain with, for example, multivariate logistic analysis.

\section{Practical implications and original value}

The results are especially interesting for institutions responsible for the management of public or private aid programs aiming to foster entrepreneurship. They offer a guide for the assessment of new entrepreneur projects, taking into account variables or conditions that are particularly relevant to predict survival probabilities, at least at the short and medium run.

This study highlights the fact that the entrepreneur's background should be object of consideration due to two reasons. Firstly, because it should be taken to account when deciding the projects to bet on, and, secondly, because theoretical and practical training of actual and potential entrepreneurs is shown as a profitable investment of public and private funds, especially in the case of social ventures. Finally, a real and flexible social venture plan could be a very interesting management tool, if managers have the knowledge and the will for an adequate implementation.

KEYWORDS: Social entrepreneurship, business survival, entrepreneur characteristics, size, business plan, fsQCA. 


\section{Introducción' 1}

Frente al emprendimiento de negocios, existe otro tipo de emprendimiento que la literatura ha bautizado como "social", que tiene como objetivo la creación de valor social sostenible, priorizando la creación de valor sobre la apropiación del mismo (Santos, 2012). Este emprendimiento no sólo constituye un fenómeno en clara expansión (Bosma y Levie, 2009), sino que está en el origen del desarrollo de nuevas industrias, a la vez que permite la validación de nuevos modelos de negocio y dedica recursos a problemas sociales olvidados, tanto por el Estado como por el mercado (Baglioni, 2017; Bornstein y Davis, 2010). Asimismo, resulta muy efectivo en lo que se refiere a la creación de empleo (Harding, 2004). Por todo ello, el emprendimiento social ha sido reconocido por diferentes autores como una contribución para la sociedad (Alvord et al. 2004, Dees 2001, Drayton 2002, Peredo y McLean 2006 , Zahra et al. 2009).

Por todo lo anterior, resulta de especial interés el análisis de los factores de éxito o fracaso de las empresas sociales (Simón et al. 2012; Zahra et al, 2009). Si bien supervivencia y éxito, o cierre y fracaso, no pueden considerarse sinónimos, resulta innegable la relevancia del análisis de la supervivencia de las empresas de reciente creación, cuyas tasas de mortalidad son muy elevadas durante sus primeros años de vida (Head, 2003).

Tradicionalmente, la literatura ha destacado la importancia de tres tipos de variables, relacionadas con las características del emprendedor, del negocio y los factores del entorno (Franco y Haase, 2010; Schutjens y Wever, 2000). Este trabajo se centra en analizar relaciones causales entre las diferentes combinaciones posibles de: a) características del emprendedor (formación, experiencia y motivación para emprender), y b) características del negocio (capital inicial, plantilla inicial y calidad del proyecto de empresa, evaluado por su viabilidad organizativa, económica y financiera); y la supervivencia de las empresas sociales.

Las proposiciones o preceptos susceptibles de ser testados en el trabajo pueden resumirse en que ninguna de las características o condiciones consideradas puede, por sí misma, garantizar la supervivencia; que un buen proyecto no es garantía de nada; y que las probabilidades de éxito aumentan cuando este se combina con un emprendedor vocacional, con formación y experiencia afín y unos recursos iniciales superiores a la media.

La supervivencia se analiza al final del tercer y sexto año posterior al de su creación. La muestra se compone de 291 empresas para el estudio en $T+3$ y 212 para el estudio en $T+6$, tras eliminar las

1.- Esta investigación ha sido financiada con fondos del proyecto UV-INV-AE15-348043, de la Universidad de Valencia. 
empresas desaparecidas durante el primer periodo. Todas estas empresas, pertenecientes a diferentes sectores de actividad, fueron creadas entre 2001 y 2005 en el ámbito de la Comunidad Autónoma Valenciana.

Para el análisis de los resultados se ha utilizado el software fsQCA 2.5 (Ragin y Davey, 2014). El Análisis Cualitativo Comparativo, específicamente el fsQCA, presenta algunas diferencias y ventajas respecto a los métodos cuantitativos tradicionales pues estudia los casos como configuraciones de características y condiciones, causalmente relevantes, y un resultado, en nuestro caso, la supervivencia. El fsQCA admite que diferentes condiciones pueden llevar a un mismo resultado (Fiss, 2011; Woodside y Zhang, 2013).

Los resultados muestran que ninguna de las condiciones analizadas es necesaria para la supervivencia empresarial de las empresas sociales en los dos periodos analizados. Tampoco existe un único patrón para la supervivencia de las empresas ni en $t+3$ ni en $t+6$. Concretamente, existen 4 patrones causales que conducen a la supervivencia en ambos momentos del tiempo, si bien existen algunos cambios en cuanto a las condiciones que se revelan como más determinantes de la supervivencia en cada periodo.

En el siguiente apartado se plantea el marco teórico en el cual se define el emprendedor social y se revisan los principales factores condicionantes de la supervivencia para fundamentar una serie de proposiciones o preceptos a validar respecto a su relevancia. En el apartado metodológico se justica la idoneidad del análisis cualitativo comparativo y se explica el procedimiento a seguir, se caracteriza la muestra, se definen los condicionantes causales y las variables de resultados y se procede a la calibración de los datos originales. El siguiente apartado recoge los resultados de los análisis de necesidad y suficiencia. Por último, se presentan las conclusiones, implicaciones prácticas y la sugerencia de futuras líneas de investigación.

\section{Marco teórico}

\subsection{Definiendo el emprendimiento social}

Desde un punto de vista histórico y una perspectiva amplia, el emprendimiento social siempre ha existido como reflejo de iniciativas impulsadas por la sociedad civil, en las que se han puesto en común recursos para satisfacer una necesidad social compartida (Fournier, 2011). No obstante, el emprendimiento social como fenómeno reconocido y organizado, nace en las últimas décadas del siglo XX. Si bien, a partir de ese momento, se ha destacado la importancia del emprendimiento social desde 
la perspectiva de su impacto económico y social, y es patente el interés de la academia por el fenómeno, todavía no existe un amplio consenso en relación a su definición (Acs et al., 2013; Baglioni, 2017; Light, 2006). De hecho, existen diferentes escuelas de pensamiento, entre las que se encentran ciertas especificidades, especialmente entre los enfoques europeo y estadounidense (Defourny y Nissens, 2012 y 2017) (ver cuadro 1).

\section{Cuadro 1. Escuelas de pensamiento sobre emprendimiento social}

\begin{tabular}{|l|l|l|}
\hline \multicolumn{2}{|l|}{ EEUU } \\
\hline $\begin{array}{l}\text { Escuela de "gene- } \\
\text { ración de ingresos" }\end{array}$ & $\begin{array}{l}\text { Enfoque comercial } \\
\text { nonprofit }\end{array}$ & $\begin{array}{l}\text { - Impulsada por organizaciones como la Social Enterprise Alliance. } \\
\text { - Alude a la utilización de actividades comerciales por parte de organizacio- } \\
\text { nes nonprofit como apoyo a su misión social. } \\
\text { - El objetivo en conseguir mejor financiación, garantizando la sostenibilidad. }\end{array}$ \\
\hline onfoque de negocio \\
& & $\begin{array}{l}\text { - Abarca todas las formas de iniciativas comerciales, no solo las impulsados } \\
\text { por organizaciones nonprofit, incluyendo las empresas con ánimo de lucro, } \\
\text { siempre que negocien con un fin social. } \\
\text { - El negocio no solo constituye un medio para conseguir mejor financiación } \\
\text { sino, un medio para conseguir una mayor eficacia. }\end{array}$ \\
\hline Escuela de "innovación social" & $\begin{array}{l}\text { - Impulsada por organizaciones como ASHOKA. } \\
\text { - Pone el énfasis en el perfil y comportamientos del emprendedor. } \\
\text { - El emprendedor social es agente de cambio que busca "nuevas combina- } \\
\text { ciones" para crear valor social (nuevos servicios, nuevos niveles de calidad, } \\
\text { nuevos métodos de producción, nuevas formas de organización o nuevos } \\
\text { mercados. } \\
\text { - Destaca la naturaleza sistémica de la innovación y su impacto en un amplio } \\
\text { plano social. } \\
\text { - Trabajos recientes admiten oportunidad de innovación social emprendedora } \\
\text { impulsada fuera del sector nonprofit. }\end{array}$ \\
\hline
\end{tabular}




\begin{tabular}{|l|l|}
\hline EUROPA \\
\hline \\
\hline - Concepto muy vinculado a la Economía Social, pues la mayor parte de las empresas sociales per- \\
tenecen o tienen su origen en el tercer sector. Tienen especial impacto en el ámbito de la inte- \\
gración laboral y los servicios personales. Un primer hito importante es la creación de la \\
"cooperativa social" en Italia, que ejemplifica el denominado Social Cooperative Model. \\
- Le suceden otras iniciativas en Francia, Portugal, España y Grecia, que también desarrollan nue- \\
vas formas legales de tipo cooperativo. Bélgica, Reino Unido e Italia (nueva norma) establecen \\
modelos de empresa social más abiertos a otras formas organizativas. Se exige intención social \\
explícita y que no se dediquen al enriquecimiento de sus miembros, sino que sus excedentes se \\
reinviertan principalmente en el negocio o la comunidad. Estas nuevas formas organizativas y \\
modelos de empresas sociales pueden encuadrarse en el Social Business Model. \\
- Tampoco se puede obviar la existencia de iniciativas sociales empresariales promovidas por el \\
Sector Público, buscando reducir costes y mejorar la eficiencia (Public Sector Social Enterprise \\
Model). \\
- La Red Europea de Investigación EMES juega un papel esencial en su estudio conceptual y empí- \\
rico y establece indicadores de un "tipo ideal" de empresa social: \\
* Actividad continua de producción de bienes y servicios \\
* Nivel significativo de riesgo económico \\
* Mínima cantidad de trabajo remunerado \\
* Objetivo explícito para beneficiar la comunidad \\
* Iniciativa lanzada por ciudadanos u organizaciones de la sociedad civil \\
* Distribución de beneficios limitada \\
* Alto grado de autonomía \\
* Facultad de decisión no basada en la propiedad de capital \\
* Naturaleza participativa
\end{tabular}

FUENTE: Elaboración propia a partir de Defourny y Nissens (2012 y 2017).

El hecho de que el emprendimiento social incluya una variedad de realidades dificulta el que pueda definirse con precisión y lograr el deseado consenso. El modelo americano no pone el énfasis del europeo en las formas organizativas como modo de asegurar la persecución de la misión social, ni exige explícitamente dinámicas colectivas (destacan la importancia del perfil individual del emprendedor social y su papel central) aunque si son compatibles con su modelo de empresa social (Defourny y Nissens, 2012). Desde una óptica europea, juega un papel esencial el emprendedor social, como emprendedor colectivo, en el que suele además recaer e doble rol de trabajado y socio o propietario de la empresa (Ciruela et al., 2016).

Pueden encontrarse como elementos comunes a las definiciones más relevantes, su caracterización como actividad innovadora, promovida por la sociedad civil, productora de bienes y servicios, cuyos ingresos pueden provenir de diversas fuentes que incluyen donaciones y subvenciones, pero también una actividad comercial sujeta a riesgos de mercado, que puede combinar trabajo retribuido y voluntariado y que tiene una clara orientación a la creación de valor social, en la que el beneficio económico, en su caso, juega un papel instrumental y financiero. 
Respecto a la innovación, el emprendedor social constituye uno de los principales agentes de innovación social, concepto sobre el cual todavía no existe consenso, pero para el que pueden establecerse una serie de elementos clave: satisfacer necesidades humanas no atendidas, originando cambios en las relaciones sociales, incrementando los niveles de participación, mejorando la capacidad sociopolítica y el acceso a los recursos necesarios de la ciudadanía (Hernández-Ascanio et al., 2016). Su naturaleza participativa ha sido señalada por autores como Peraza et al. (2016) como un factor susceptible de favorecer la innovación. En todo caso, resulta indudable que una iniciativa social puede ofrecer algo que el mercado aún no está ofreciendo, de hecho, suele actuar en aquellos espacios desatendidos por Estado y Mercado (Austin et al. 2006). Además, puede hacerlo con un tipo de organización totalmente innovadora y abrirse a nuevos mercados/sociedades.

La creación de valor social constituye el fundamento del emprendimiento social. Desde una perspectiva restrictiva, algunos autores consideran que el lucro es incompatible con el emprendimiento social, restringiendo su ámbito de actuación a las organizaciones no lucrativas (Harding, 2004; Thompson, 2002). Otros autores, como Arthur et al., (2006) consideran que la creación de valor social no es incompatible con el lucro, pero tampoco es condición necesaria la existencia del mismo. Autores como Dorado (2006) y Drayton (2002) señalan que tanto el emprendimiento de negocios como el social intentan tener un rendimiento económico y que pueden tener beneficios. En este caso, como señala Beugré (2011) la creación de valor económico sería sólo un medio para hacer a la empresa eficiente y autosuficiente. Esta última es la tesis mayoritaria y la literatura ofrece múltiples ejemplos de empresas orientadas a la creación de un valor social que tienen beneficios financieros (Van Slyke y Newman, 2006). Según Kachlami (2016) el crecimiento del neoliberalismo ha llevado a cierta hibridación del sector social, por la introducción de una lógica de mercado que conlleva alcanzar la doble misión de crear valor social, consiguiendo la sostenibilidad social, algo que no deja de generar ciertas tensiones en la empresa (Chandra, 2017) y posicionamientos encontrados en la literatura.

Lo característico del emprendedor social, es el énfasis que pone en la creación de valor en todas sus formas, frente al énfasis del emprendedor de negocios en la apropiación de ese valor (Santos, 2012). La creación de valor supone un aumento de la utilidad social, una vez descontados los recursos utilizados en la actividad, mientras que la apropiación de valor implica que el emprendedor es capaz de capturar una parte del valor creado mediante la actividad (Mizic y Jacobson, 2003). Si bien la creación de valor es imprescindible para garantizar una apropiación de valor sostenible, y cierta apropiación de valor es imprescindible para asegurar la sostenibilidad y crecimiento de las organizaciones, estas pueden llevar a cabo acciones que pueden incrementar el potencial de apropiación de valor en detrimento de la creación de valor. Por tanto, existe una relación de intercambio entre creación y apropiación de valor y las organizaciones deben elegir dónde poner el énfasis, elección que condicionará su naturaleza y su definición como emprendedor social o de negocios (Santos, 2012).

Para autores como Dees (2001) y Lepoutre et al. (2011), las claves para diferenciar el emprendedor social del comercial o de negocios y definir los distintos tipos de emprendimiento social se encuentran en tres pilares: a) su misión que prioriza un fin social (o medioambiental) frente al fin eco- 
nómico; b) el énfasis en la innovación, pues el emprendedor social debe participar en el desarrollo de soluciones creativas y diferentes; y c) los métodos de financiación, que pueden recursos públicos 0 donaciones, con los recursos económicos que genera su propia actividad. En función de estos tres pilares Lepoutre et al. (2011), diferencian entre los siguientes tipos de emprendimiento social:

1. ONG tradicional: el objetivo social/medioambiental es el único o principal. Estatus de organización sin ánimo de lucro. Más del 95\% de sus ingresos dependen de donantes. No presentan ningún tipo de innovación esencial.

2. Empresa social sin ánimo de lucro: el objetivo social/medioambiental es el único o principal. Estatus de organización sin ánimo de lucro. Más del $67 \%$ de sus ingresos dependen de donantes. Es innovadora en sus proyectos.

3. Empresa social híbrida: el objetivo social/medioambiental es el único u ocupa una posición muy importante. Existe una estrategia de generación de ingresos «integrada» 0 "complementaria» a la misión de la organización. Más del $5 \%$ de sus ingresos provienen del mercado.

4. Empresa social con ánimo de lucro: el objetivo social/medioambiental ocupa una posición importante pero no tiene por qué ser el único. Son innovadoras y existe una estrategia clara de generación de ingresos. Del $50 \%$ al $67 \%$ de su financiación deriva de recursos propios.

Desde esta perspectiva, el que una empresa se sitúe dentro del emprendimiento social no depende de que tenga o no ánimo de lucro, ni tampoco del modelo o tipo de organización por medio de la cual se aborda el problema social. La empresa social puede entenderse como una combinación de diferentes actores, lógicas de acción y recursos (Szimanska y Jegers, 2017)). El dictamen exploratorio del CESE (2011), reconoce explícitamente la pluralidad de formas y de modelos jurídicos en los cuales se plasma el emprendimiento social, así como su estrecha vinculación a la economía social. A este respecto Monzón (2016: 302), enmarca la empresa social "en el ámbito claustral de la economía social", en sintonía con la iniciativa en favor del emprendimiento social de la Comisión Europea de 2011. En esta misma línea, Picciotti (2017) considera las empresas sociales como las organizaciones que juegan un rol emprendedor en el marco de la economía social.

El informe del GEM (2009) concluye que los tipos de organizaciones creadas y los problemas que se pretenden atajar son muy variados. En general, en los países menos desarrollados, los emprendedores sociales se preocupan especialmente por cubrir necesidades básicas, como pueden ser la sanidad, el acceso al agua y su salubridad o el apoyo a actividades agrícolas de zonas rurales. En países más desarrollados, se centran prioritariamente en "dar apoyo a la cultura, proveer servicios a las personas discapacitadas, colaborar en procesos de recogida de residuos y protección del medioambiente u ofrecer actividades gratuitas como puede ser el networking social" (Curto, 2012: 15).

\subsection{Condicionantes de la supervivencia}

Resulta especialmente relevante el análisis de las características de los emprendedores y de las empresas sociales que más impacto tienen sobre sus resultados y sus probabilidades de supervi- 


\section{SUPERVIVENCIA DE EMPRESAS SOCIALES DE NUEVA CREACIÓN. UN ENFOQUE BASADO EN EL ANÁLISIS CUALITATIVO COMPARATIVO FSQCA}

vencia. En este sentido, Haugh (2007) y Zahra et al. (2009) señalan la necesidad imperiosa de investigar en relación a los factores de éxito o fracaso de las empresas sociales. No obstante, aun en la actualidad siguen siendo muy escasos los trabajos dedicados a esta cuestión.

Especial interés merece este estudio en el caso de las empresas de reciente creación, pues existe una amplia evidencia empírica de que éstas presentan unas tasas de cierre altas y significativamente más elevadas que las empresas consolidadas (Bruderl y Schussler 1990; Simón-Moya et al. 2012; Simón-Moya y Revuelto-Taboada, 2016). Si bien autores como Head (2003) aconsejan distinguir entre cierre y fracaso, el análisis de supervivencia, opción elegida en el presente trabajo, tiene muchos precedentes en la literatura que considera a la supervivencia como uno de los indicadores más relevantes para determinar el éxito de una empresa (Brüderl y Schüssler, 1990; Cooper, et al. 1994; Haber y Reichel, 2005).

Tradicionalmente, la literatura ha destacado la importancia de tres tipos de factores que influyen en el éxito y supervivencia de las empresas, relacionados con las características del emprendedor, con las características del negocio y los factores externos o del entorno (Franco y Haase, 2010; Schutjens y Wever, 2000). A este respecto, investigaciones previas no han encontrado evidencia de diferencias sustanciales respecto al papel que juegan estos factores entre empresas de negocios y sociales (Simón-Moya et al. 2012). En este caso nos centraremos en los dos primeros bloques de factores, considerando además el plan de negocios o empresa, que puede considerarse un factor a caballo entre los dos bloques, pues supone, en cierto modo un "recurso" de la empresa, cuya formulación e implantación depende, de forma sustancial, del emprendedor. Obviaremos el tercer bloque, conscientes de que supone una limitación del presente trabajo, porque todas las empresas consideradas fueron creadas en un ámbito geográfico muy delimitado, así como por la imposibilidad de evaluar las especificidades del sector concreto al que pertenece cada una de las empresas creadas.

\subsubsection{Características del emprendedor}

Si bien se ha avanzado en la definición del emprendedor social, coexisten diversas concepciones y son escasos los estudios que abordan las características del mismo que tienen un impacto significativo sobre las probabilidades de supervivencia de la empresa social. Dess et al. (2001) lo definen como agente de cambio, que adopta una misión orientada a la creación de valor social de forma sostenible, capaz de reconocer nuevas oportunidades para lograr la misión, comprometido con la innovación continua, el aprendizaje y la adaptación, que actúa con convicción y exhibe un gran sentido de transparencia, rendición de cuentas y verificación de los resultados. Roberts y Woods (2005), nos hablan de individuos visionarios, apasionadamente dedicados, que identifican/construyen, evalúan y persiguen oportunidades para el cambio social transformativo. 


\section{Cuadro 2. Resumen de características del emprendedor social}

\begin{tabular}{|l|l|}
\hline Emprendedor con capacidad de gestión & $\begin{array}{l}\text { ejecutivos sin fines de lucro (Boschee, 1998); calidad empresarial (Drayton, 2002); } \\
\text { cualidades y comportamientos que asociamos con el empresario (Thompson, } \\
\text { 2002); gente de negocios (Roberts y Woods, 2005); capacidad para reconocer y } \\
\text { aprovechar oportunidades (Peredo y McLean, 2006); aplican estrategias de nego- } \\
\text { cio Sharir y Lerner, 2006); líderes (Brouard y Larivet, 2010). }\end{array}$ \\
\hline Ética y valores & $\begin{array}{l}\text { equilibran imperativos morales y el afán de lucro (Boschee, 1998); fibra ética } \\
\text { (Bornstein \& Davis, 2010; Drayton, 2002); fuertes valores sociales (Austin et al., } \\
\text { 2006). }\end{array}$ \\
\hline Innovador/Agente de cambio & $\begin{array}{l}\text { agente de cambio (Dees, 2001; Sharir y Lerner, 2006; Brouard y Larivet, 2010); } \\
\text { crean soluciones innovadoras (Alvord et al., 2004); personas o grupos que apor- } \\
\text { tan innovación (Peredo y McLean, 2006); cambio social a través de ideas inno- } \\
\text { vadoras (Light, 2006). }\end{array}$ \\
\hline Asunción de riesgos & $\begin{array}{l}\text { capacidad para asumir riesgos (Brinckerhoff, 2000); aceptan el riesgo (Peredo y } \\
\text { McLean, 2006); aversión al riesgo por debajo de la media (Curto, 2012). }\end{array}$ \\
\hline
\end{tabular}

FUENTE: Elaboración propia a partir de Nicolás-Martinez (2014).

Los emprendedores sociales, no actúan necesariamente motivados por remuneraciones materiales o monetarias, si no que les mueve una motivación prosocial (Grant, 2008; Penner et al., 2005). Fournier (2011) señala que el emprendedor social debe aliar creatividad y pragmatismo, y que se diferencia con el emprendedor de negocios en que es un agente de cambio social más que de cambio económico, y en que su motivación y misión son diferentes. Asimismo, señala que debe tener atributos de líder, visión, con un alto grado de ética y una aversión al riesgo por debajo de la media (Enciso et al., 2012). Nicolás-Martinez (2014) ofrece una interesante panorámica de definiciones del emprendedor social (individual o colectivo), que destacan algunas características esenciales, al margen de la ineludible misión social, que resumimos en el cuadro $\mathrm{n}^{0} 2$ de la página anterior.

En definitiva, reconociendo ciertas peculiaridades del emprendedor social, resultan a priori perfectamente asimilables las relaciones establecidas, en el ámbito del emprendimiento de negocios, entre determinadas características del emprendedor y el éxito del emprendimiento. Como señala Dess (1998), es imprescindible que el emprendedor social reconozca y aproveche las oportunidades y tanto la formación previa como la experiencia actúan como potenciadores de esta detección y facilitan un mejor aprovechamiento de la misma. Simón-Moya et al. (2015), en un estudio exploratorio de carácter cualitativo señalan como factores determinantes para activar la alerta social (capacidad para detectar fallos en el mercado y descubrir oportunidades) la cercanía al foco del problema social, la cercanía a emprendedores y la formación y experiencia previas. 


\section{SUPERVIVENCIA DE EMPRESAS SOCIALES DE NUEVA CREACIÓN. UN ENFOQUE BASADO EN EL ANÁLISIS CUALITATIVO COMPARATIVO FSQCA}

Fournier (2011) también señala la importancia de los conocimientos y experiencias del emprendedor social, así como de su capacidad para crear nuevas prácticas a partir de las prácticas del sector tradicional. La evolución del emprendimiento social ha llevado a modelos de negocio cada vez más complejos e innovadores, que exigen un elevado nivel de capacitación técnica y que pueden valerse de la experiencia previa en el sector 0, cuanto menos, en tareas de gestión. La Schwab Foundation señala que los emprendedores sociales persiguen aliviar la pobreza con celo emprendedor, métodos empresariales y el coraje de innovar y superar las prácticas tradicionales (Enciso et al., 2012). Ello es especialmente relevante en un contexto social en el cual resulta más difícil acceder a recursos financieros y atraer, retener y motivar recursos humanos valiosos, ante la dificultad para ofrecer compensaciones económicas competitivas (Austin et al., 2006; Fournier, 2011).

Tanto la formación genérica, como la más específica parecen tener un impacto positivo sobre el éxito del emprendimiento. La primera, porque le capacita para transformar información en "saber hacer". La específica, que se centra en procesos, tecnologías o productos relevantes en el sector, permite al emprendedor detectar mejor las necesidades de sus "clientes, usuarios o beneficiarios", utilizar los recursos de forma más eficiente y reducir costes (Castrogiovanni, 1996). Ciruela et al. (2016), RibeiroSoriano y Castrogiovanni, (2012) y Van Praag (2003), destacan la importancia de la formación específica para el negocio, así como la experiencia afín al mismo. La mayoría de los autores coinciden en que la experiencia en el sector y/o como emprendedor constituye un factor relevante para el éxito del emprendimiento (Luk 1996; Reuber y Fisher 1999).

La literatura también ha puesto un énfasis considerable en la importancia de la motivación del emprendedor (Headd, 2003; Van Praag, 2003). En general, se diferencia entre dos tipos de motivación: oportunidad y necesidad. Los emprendedores por oportunidad buscan autonomía e independencia, son emprendedores vocacionales que inician su actividad al detectar una oportunidad de negocio (Shane y Vankataraman, 2000). En el caso del emprendedor social las oportunidades se presentan como soluciones a problemas sociales (Austin et al. 2006) debidos, en general, a fallos del mercado (Kirzner, 1979). Los emprendedores por necesidad no tienen especial interés en el negocio en sí mismo, inician la actividad para evitar el desempleo o el empleo precario. Si bien este tipo de emprendedor es especialmente característico del sector business, algunas investigaciones muestran como, al margen de la motivación prosocial que puedan tener, muchas iniciativas de emprendimiento social surgen como una vía para evitar la inactividad, trabajando en un sector que representa valores compartidos por el emprendedor (Fernández-Guerrero y Revuelto-Taboada, 2009). En cualquier caso, la motivación sería seguramente mixta y dado que en el presente trabajo el emprendedor social por necesidad se define como tal cuando emprende desde una situación de desempleo o precariedad laboral, esta dicotomía resulta asumible.

La literatura sugiere que los emprendedores por oportunidad obtienen mejores resultados y sus empresas sobreviven más que las creadas por emprendedores por necesidad (Headd 2003; Ho y Wong 2007; Van Praag 2003). Asimismo, señala que su mayor capacidad de innovación y compromiso cons- 
tituyen factores importantes a la hora de explicar estos resultados (El Harbi y Anderson 2010; Ho y Wong, 2007). A menudo los emprendedores por necesidad buscan obtener ingresos con urgencia y cuentan con una escasa capacidad de inversión, por lo que suelen constituir empresas de menor tamaño. También tienden a tener un menor nivel de formación que los emprendedores por oportunidad (Kelley et al. 2011). En definitiva, las características de los emprendedores por necesidad podrían explicar las diferencias en las ratios de supervivencia con los emprendedores por oportunidad obtenidas en estudios previos.

En todo caso, consideramos especialmente relevante destacar cómo pueden existir diferentes combinaciones de condiciones, que pueden llevar a resultados similares. Respecto a lo señalado en el párrafo anterior, un emprendedor por necesidad, pero con formación, experiencia y recursos financieros y plantilla suficiente, sí podría tener altas probabilidades de éxito. Por ello, consideramos más relevante estudiar el impacto sobre la supervivencia de diferentes combinaciones de condicionantes causales que analizar cada uno por separado.

\subsubsection{Características de la empresa}

Una empresa de reciente creación tiene más probabilidades de fracasar, como ya señalaron Brüderl y Schüssler (1990) al plantear lo que denominaron "lastre o desventaja de novedad". Centrándose en las empresas sociales, diversos trabajos señalan que aproximadamente el $40 \%$ de las iniciativas sociales no superaban los 5 años tras su fundación (Goodson et al, 2001; Steadman et al., 2002). Otros estudios más recientes señalan una alta mortalidad de las empresas sociales, no habiendo encontrado diferencias significativas en los niveles de supervivencia en los primeros años de vida entre empresas sociales y comerciales o de negocios (Fernández-Guerrero y RevueltoTaboada, 2009; Simón-Moya, et al., 2012). Por el contrario, un estudio exploratorio que comparó la supervivencia de las 100 mayores empresas sociales y las 100 mayores empresas comerciales del Reino Unido entre 1984 y 2014, concluyó que las primeras mostraron un mayor nivel de supervivencia (Denny, 2014). Cantarero et. al (2017) y Gimmon y Spiro (2013) también señalan que las empresas sociales muestran ventajas sobre las comerciales en cuanto a su supervivencia.

En todo caso, los altos niveles de mortalidad en ambos tipos de empresas son evidentes, especialmente, en las primeras etapas de su existencia, incluso cuando se trata de iniciativas con apoyo de instituciones públicas (Chandra y Wong, 2016). La teoría de la infant industry explica las implicaciones de esta desventaja, o lastre, indicando que enfrentan unas barreras de entrada que puede precipitar su fracaso, pues permiten a las empresas establecidas gozar de ciertas ventajas competitivas (Kerr y Nanda, 2011; Masa et al., 2016). Una de las principales barreras deriva de la dificultad para acceder a recursos de capital, pero también puede ser limitado su acceso a capital humano especializado pues contará, en general, con escasos recursos para adquirirlo, y ha gozado de escaso tiempo para desarrollarlo internamente. 
Este problema de escasez de recursos resulta especialmente significativo en el caso de las empresas sociales (Austin et al., 2006; Fournier, 2011). Su capacidad para atraer capital depende, fundamentalmente, de su solvencia y su capacidad de pago, intrínsecamente vinculadas a su capacidad para generar ingresos, por lo que su carácter de entidades no orientadas al lucro reduce sustancialmente sus posibilidades de acceder a crédito en buenas condiciones. Estas entidades dependen, en gran medida, de la aportación inicial de los promotores, subvenciones y donaciones pues, si bien es cierto que han ido apareciendo entidades más proclives a financiar empresas sociales, como la banca ética y algunas fundaciones filantrópicas de capital riesgo, y algunos gobiernos han lanzado algunos programas específicos de financiación del emprendimiento social, su impacto es reducido (NicolásMartinez, 2014).

Por lo que respecta al capital humano, en general, las empresas sociales no pueden (ni muchas veces quieren), ofrecer salarios competitivos, por lo que dependen más que otro tipo de organizaciones de la satisfacción personal que puede sentir los empleados por el cumplimiento de la misión social, y del voluntariado. De hecho, en muchos casos, la actividad profesional en la empresa social constituye una actividad secundaria en la vida profesional del colaborador (Cornelius et al., 2010; NicolásMartinez, 2014).

Por otro lado, Stinchcombe (1965) señala que cada empresa necesita un periodo para desarrollar nuevos roles y desarrollar relaciones con el entorno, especialmente con consumidores, proveedores, beneficiarios, financiadores, y demás instituciones públicas y privadas, con las cuales han de relacionarse con motivo de la actividad emprendida. Además, su menor conocimiento del entorno les lleva, en algunos casos, a procesos de "prueba y error" (Starr y MacMillan, 1990) reduciendo su eficiencia. Realidad esta, tan aplicable al caso del emprendimiento social, como al de negocios.

Otro "lastre" bien conocido, muy común en el caso de las empresas de reciente creación y relacionada con la anterior, es el "lastre o desventaja de pequeñez" (Brüderl y Schüssler, 1990). Las evidencias empíricas muestran que el tamaño medio de las iniciativas de emprendimiento social es muy pequeño y su crecimiento escaso en sus primeros años de vida (Fernández-Guerrero y RevueltoTaboada, 2009). El agravante del tamaño supone, generalmente, una desventaja frente a otras de mayor dimensión, especialmente cuando se trata de empresas ya establecidas que pueden gozar de economías de escala, además de un mayor efecto experiencia y, en general, un acceso más fácil a recursos financieros (Masa et al., 2016). En relación con el tamaño, dos de los factores que muestran una relación más consistente y positiva con el éxito y la supervivencia empresarial son el número de empleados (López-García y Puente, 2006) y el capital inicial (Brüderl, et al. 1992; Headd, 2003; Schutjens y Wever, 2000).

Ahora bien, abundando en lo ya comentado respecto a las combinaciones de factores relacionados con las características del emprendedor, contar con un mayor capital inicial y mayor plantilla, puede tener un efecto positivo sobre la supervivencia, especialmente en la primera fase de vida de una 
empresa. No obstante, en ausencia de un emprendedor con el perfil adecuado, la empresa se verá, seguramente, abocada al fracaso. Por el contrario, un emprendedor por oportunidad con un buen nivel de formación y experiencia afín tendrá probablemente, igual o más éxito que otro tipo de emprendedor pese a contar con menores recursos inicialmente. La literatura sobre "bricolage" (Baker y Nelson, 2005; Senyard et al. 2014), ofrece argumentos bastante convincentes a este respecto cuando señala la capacidad de ciertos emprendedores enfrentados a situaciones de severas restricciones de recursos disponibles, para poner en marcha proyectos innovadores. La capacidad del emprendedor para recombinar recursos aparentemente obsoletos y de escasa utilidad, puede hacerle conseguir ventajas inesperadas (Garud y Karnoe, 2003). Los conocimientos y habilidades del emprendedor le permiten detectar todo del rango de posibles usos de los recursos existentes (Di Domenico et al., 2010) lo cual, combinado con las indispensables dosis de creatividad, condición necesaria, aunque no suficiente, para la innovación (Amabile et al, 1996), pueden llevarle al éxito donde otros fracasaron contando con mayor dotación inicial de recursos.

Ahora bien, la utilización de esta capacidad de aprovechamiento y recombinación de recursos escasos, no solo no es incompatible con la elaboración de un plan de "negocio" o de empresa, entendido en un sentido amplio y, por tanto, aplicable a las iniciativas sociales, que utilizan procesos de operaciones internos y recursos similares a las empresas comerciales (Gimmon y Spiro, 2013; Meyckens et al., 2010). De hecho, como veremos en el siguiente apartado, la elaboración del plan puede ofrecer variados beneficios a la iniciativa social.

\subsubsection{El plan de la empresa social}

Como señala Varela (2001) el plan de empresa constituye un mecanismo para proyectar la empresa en el futuro, prever dificultades e identificar posibles soluciones a las coyunturas que pudieran presentarse, permite reducir los riesgos del proyecto al tomar decisiones con más información y de mejor calidad, facilitando además la conexión de la empresa con su entorno. El plan debe desarrollar los procedimientos y estrategias necesarias para convertir una oportunidad en una realidad y debe constituir un punto de referencia para medir el desempeño de la empresa durante su implementación, al incluir proyecciones financieras, de gestión, sociales y legales (Sapag y Sapag, 2003).

No obstante, los trabajos que han analizado el impacto de la existencia de un plan de empresa sobre las probabilidades de éxito de la misma, se han centrado casi exclusivamente en el emprendimiento de negocios y ofrecen evidencia contradictoria (Conway, 2008). Por un lado, los trabajos de Busstra y Verhoef (1993) y Henninger et al. (1993) concluyen que contar con un plan de empresa no es una garantía de éxito. Reid y Smith (2000) por su parte concluyen que el contar con un plan de empresa no afecta positivamente el rendimiento de los nuevos proyectos empresariales, pero sí que aumenta el número de meses en que los directivos proyectan los efectos de sus decisiones a la hora de evaluar su impacto. Sandberg et al. (2001) indicaron que en la mayoría de los casos se concedía una gran importancia al plan de empresa fundamentalmente para conseguir recursos financieros, sin embargo, consideraban que el plan de empresa tenía un valor mínimo una vez se han recibido los 


\section{SUPERVIVENCIA DE EMPRESAS SOCIALES DE NUEVA CREACIÓN. UN ENFOQUE BASADO EN EL ANÁLISIS CUALITATIVO COMPARATIVO FSQCA}

fondos. Honig y Karlsson (2004) nos muestran que la adopción del plan deriva de presiones institucionales coercitivas y miméticas y que las empresas de reciente creación que utilizan planes de empresa formales no muestran mayores niveles de supervivencia ni una mayor rentabilidad.

Por el contrario, Brüderl et al. (1992) consideran que el plan sí tiene una influencia positiva sobre las probabilidades de éxito. Orser et al. (2000) encontraron que la presencia de un plan de empresa estaba altamente correlacionada con mejoras en los ingresos. Perry (2001), tras analizar una muestra de empresas de la base de datos Duns and Bradstreet, concluyó que las empresas que entraban en bancarrota utilizaban menos la planificación formal. Stewart (2002) concluye que, en el caso de los pequeños negocios con orientación internacional el contar con un plan de empresa formal contribuye efectivamente al éxito de este tipo de negocios. Delmar y Shane (2003) señalan que, el hecho de disponer de un plan de empresa formal, aumenta la probabilidad de recibir capital externo, facilitan el desarrollo del producto y la adecuada organización de la compañía, aumentando sus probabilidades de supervivencia. Lussier y Halabi (2010) validaron en su trabajo la hipótesis de que las empresas que no los desarrollan tienen más probabilidades de fracaso.

Conway (2008), señala que los escasos trabajos que han estudiado los procesos de planificación de las empresas sociales, indican que sus directivos han mostrado experiencias variadas a la hora de diseñar y aplicar técnicas de planificación de negocios, mostrando, a menudo, bajos niveles de sofisticación (Chapman et al, 2007). Ciruela et al. (2016) destacan la importancia de la planificación de la actividad y la fijación de objetivos y estrategias para el éxito de las sociedades laborales, no solo desde el prisma de la elaboración del plan de empresa, sino de la conciencia real de los fundadores de la necesidad de establecer pautas al respecto. Los planes de negocio de las empresas sociales pueden proporcionarles diversos beneficios: claridad de dirección, conocimiento del mercado y de la propia empresa, identificación de futuros retos, mayor compromiso de los stakeholders, atracción de inversiones y financiación, etc. (Conway, 2008; Scott, 2001). Scott (2001: 62) lo define como "el cimiento de una dirección disciplinada y efectiva" de las empresas sociales. Ahora bien, estas empresas suelen presentar grandes limitaciones en finanzas, marketing, capacidad de análisis y planificación (Thompson, 2002), por lo que suelen necesitar ayuda externa (lo que no siempre les resulta fácil obtener) si quieren cubrir estas deficiencias.

Las especiales dificultades que encuentran las empresas sociales a la hora de conseguir financiación y conseguir determinados contratos hacen más que recomendable contar con un plan de empresa, para ganar legitimidad, y demostrar no sólo que son viables, si no que sus objetivos sociales con compatibles, alcanzables y relevantes, además de medibles (Kato et al, 2017). Obviamente, el plan de una empresa social presentará especialidades en la medida en que presentan peculiaridades, como la necesidad de encajar las ideas de negocios con un propósito social claro y prioritario, una mayor dependencia de fuentes de financiación de no mercado, una gestión más democrática y la implicación de diversos tipos de stakeholders, la combinación de trabajo voluntario y retribuido, la dificultad para evaluar el valor social generado, etc. (Conway, 2008; Mastín, et al., 2017; Pearce, 2003). 
Finalmente, si nos atenemos a la literatura estratégica, la mera formulación de un plan de negocios, expresión de la estrategia pretendida o planificada, no puede considerarse una garantía de éxito. Dicho plan deberá ser correctamente implementado, utilizando los recursos y metodologías adecuadas, y su contenido constantemente adaptado en función de la evolución de las variables relevantes del entorno. Diversos autores destacan la necesidad de adoptar un proceso integrador de formación de la estrategia, racional y al mismo tiempo emergente, que combine, por un lado, la necesidad de adoptar procesos racionales y planificados y, por otro, la necesidad de flexibilidad y participación a la hora de formular e implantar estrategias en los entornos competitivos actuales (Whittington, 2006). Sin ello, las probabilidades de no sobrevivir a los retos que plantean las primeras etapas de la vida de las empresas son elevadas.

La existencia de interacciones entre diversos factores condicionantes y de relaciones causales complejas entre estos y la supervivencia empresarial, pueden estar detrás de la existencia de resultados contradictorios. A modo de ejemplo, una mayor formación y experiencia del emprendedor puede influir significativamente en la adecuada formulación, adaptación a los cambios del entorno e implementación del plan de empresa, aumentando significativamente las probabilidades de éxito. Por otro lado, cuando los emprendedores están movidos por la búsqueda de la independencia, la autonomía y la vocación de poner en marcha su propio negocio, no sólo por la necesidad de trabajar, cabe esperar que el rigor a la hora de planificar y la predisposición a llevar a cabo los esfuerzos necesarios para hacer del plan de empresa una realidad sostenible sea mayor.

Por otro lado, en la medida en que las empresas cuentan con mayor cantidad de recursos, tienen más probabilidades de sobrevivir en el periodo inicial de su existencia. Ello les puede permitir mantenerse en activo el tiempo necesario para identificar las rutinas organizativas adecuadas, aprender a colaborar con los diferentes stakeholders internos y externos, ganar legitimidad y desarrollar el conocimiento y las capacidades necesarios para una adecuada implementación del plan de negocio, así como para adaptar el contenido del mismo, sus planes y procesos en función de la evolución de las variables relevantes del entorno.

En general, los investigadores suelen analizar el impacto separado de las variables utilizadas como independientes sobre la dependiente. No obstante, son las complejas interacciones entre diferentes condicionantes, las que suelen tener un mayor impacto sobre el resultado o outcome, variable dependiente en análisis estadístico cuantitativo tradicional (Schneider et al. 2010). Por ello, consideramos pertinente analizar el efecto conjunto de los diversos factores aquí considerados sobre la supervivencia y planteamos las siguientes proposiciones o preceptos?:

2.- $A$ este respecto, no existe consenso en la literatura sobre si se puede hablar de hipótesis, proposiciones o preceptos (tenets). No obstante, en la medida en que las Ciencias del Comportamiento asocian el término hipótesis con los test estadísticos basado en las correlaciones entre variables, hemos optado por no llamarlas hipótesis. 
P1. Ninguno de los condicionantes considerados, relativos a características del emprendedor, de la empresa de nueva creación o calidad del plan de empresa, por sí solo, constituirá una condición necesaria ni suficiente 3 para la supervivencia de la nueva empresa $(t+3)$ o empresa establecida $(t+6)$.

P2. Diferentes combinaciones de los condicionantes causales o patrones causales, relativos a características del emprendedor, de la empresa de nueva creación o calidad del plan de empresa, pueden constituir condiciones suficientes para la supervivencia de las empresas de nueva creación ( $t+3)$ o empresas establecidas $(\mathrm{t}+6)$.

P3. Las combinaciones de los condicionantes causales o patrones causales, relativos a características del emprendedor, de la empresa de nueva creación o calidad del plan de empresa, que constituyen condiciones suficientes para la supervivencia, pueden variar entre empresa de reciente creación $(\mathrm{t}+3)$ y empresas establecidas $(\mathrm{t}+6)$.

\section{Metodología}

Los métodos de análisis Cualitativos Comparativos, entre los cuales se encuentra el fsQCA utilizado en el presente artículo, se han convertido en una herramienta cada vez más utilizada en el ámbito de las Ciencias Sociales, en general, y del Management, en particular. El QCA constituye un método de análisis orientado por los casos en lugar de por las variables, que permite el análisis formal y sistemático de la causalidad.

Dado que nuestras proposiciones implican la existencia de complejas interacciones entre los diversos condicionantes causales considerados, así como la posibilidad de que diversos patrones causales puedan llevar a un mismo resultado, y teniendo en cuenta las limitaciones de los métodos cuantitativos tradicionales (en este caso regresión logística) para analizar este tipo de relaciones, consideramos adecuado utilizar el QCA para testar nuestras proposiciones. Concretamente, para el análisis de los resultados se ha utilizado el software fsQCA 2.5 (Ragin y Davey, 2014).

3.- Una condición es necesaria para un resultado específico si está siempre presente cuando dicho resultado ocurre. En otras palabras, el resultado no puede ocurrir en ausencia de la condición. Una condición es suficiente para un resultado específico si dicho resultado ocurre siempre cuando esta condición está presente. Sin embargo, el resultado puede provenir también de otras condiciones, es decir, no es necesaria la presencia de dicha condición que se dé ese resultado específico. 
EI QCA presenta algunas diferencias y ventajas respecto a los métodos cuantitativos tradicionales (ANOVA, ARM, SEM), que dominan el análisis de datos en Ciencias Sociales. Estos métodos tratan cada variable tanto independiente como analíticamente distinta y separada del resto. El enfoque basado en el QCA se basa en la idea de que las relaciones causales pueden ser frecuentemente mejor entendidas en términos de relaciones entre conjuntos teóricos (set-theoretic relations) más que de correlaciones (Ragin, 2008; Ragin y Fiss, 2008). Permite, por tanto, analizar combinaciones de condicionantes causales (variables explicativas) y ofrecer resultados en situaciones en las cuales puede existir asimetría causal y equifinalidad (Fiss, 2011; Ragin, 2008; Ragin y Fiss, 2008; Schneider et al, 2010; Woodside, 2012).

El fsQCA estudia los casos como configuraciones de características y condiciones, causalmente relevantes, (antecedent conditions) y un resultado (outcome), en nuestro caso, la supervivencia. Cada configuración causal constituye una "específica combinación de ingredientes causalmente relevantes vinculados a un resultado" (Woodside y Zhang, 2013: 268). El fsQCA permite analizar todas las posibles combinaciones de condicionantes antecedentes, para testar si todas, o sólo una fracción de las condiciones causales están relacionadas con el outcome, y cómo esas condiciones relevantes deben ser combinadas, llevando a cabo un análisis de necesidad y uno de suficiencia.

Woodside (2012) señala que la suficiencia está en la esencia del método QCA. Una combinación de características y condiciones es suficiente si lleva invariablemente, o casi invariablemente, a un determinado resultado. Una condición es necesaria si está presente en todas las configuraciones causales que explican un resultado determinado. El QCA captura la idea de equifinalidad (Fiss, 2011), ya que admite que diferentes condiciones pueden llevar a un mismo resultado y que las configuraciones causales que explican un resultado determinado (supervivencia) pueden no coincidir con las que explican el resultado contrario (cierre) (Fiss, 2011; Woodside y Zhang, 2013). Además, adopta la perspectiva de que una condición simple, puede ser "necesaria", pero rara vez será "suficiente" para causar un resultado determinado 4 .

El QCA incluye una tabla de la verdad, de todas las combinaciones lógicamente posibles de condiciones antecedentes, identificando los casos que presentan cada combinación. Ello permite al investigador identificar aquellos patrones lógicamente posibles que llevan y no llevan al resultado objeto de estudio. La mayoría de los informes que utilizan la regresión múltiple no proporcionan tests minuciosos de la complejidad causal, y cuando los efectos de interacción son incluidos, su interpretación es a menudo inescrutable, especialmente cuando el número de variables independiente aumenta.

4.- Siguiendo a Schneider et al. (2010), para establecer una condición como necesaria, hemos establecido un umbral mínimo de consistencia de 0,9. Para considerar una condición como suficiente se ha establecido un umbral de consistencia de 0,8, por encima del recomendado por Ragin (2008) de 0,75. Sólo aquellas configuraciones que presenten más de 4 casos son consideradas. Estos valores de consistencia son análogos a las correlaciones estimadas en el tradicional análisis estadístico cuantitativo. 
El hecho de si las condiciones antecedentes se solapan no es una cuestión relevante en el caso del QCA. Por el contrario, la multicolinealidad de las variables independientes sí constituye un problema para métodos como la regresión múltiple, porque hace muy difícil la tarea de identificar el efecto parcial de cada variable independiente en la dependiente.

Por último, cabe señalar que, aunque el análisis QCA es aplicado, generalmente, a muestras pequeñas o medianas, matemáticamente fsQCA no tiene ningún límite en cuanto al tamaño muestral se refiere, así los análisis con fsQCA son igualmente concluyentes tanto para N-pequeña o Ngrande haciendo del fsQCA una herramienta apropiada para una amplia gama de investigaciones. Como señala Woodside (2012: 213), incluso aunque la heterogeneidad en muestras amplias es mayor "es posible descubrir implicaciones relevantes que van más allá de los descubrimientos y conclusiones que permiten los tests estadísticos con grandes muestras". Los trabajos de Feurer et al. (2015), Fiss (2011) y Woodside (2012) ilustran la eficacia del fsQCA con grandes muestras.

\subsection{Muestra y outcome o resultado a explicar}

La muestra se compone de 291 empresas para el estudio en $t+3$ y 212 para el estudio en $t+6$, tras eliminar las empresas desaparecidas durante el primer periodo. Todas fueron creadas entre $2001 \mathrm{y}$ 2005 en el ámbito de la Comunidad Autónoma Valenciana y pertenecen a diferentes sectores de actividad. Son empresas pequeñas o muy pequeñas, de hecho, muchas de ellas creadas por lo que podríamos denominar trabajadores autónomos dependientes (Navajas-Romero et al., 2017)

GEM distingue entre empresas nacientes, aquellas que llevan hasta 3 meses de actividad, nuevo emprendimiento, cuando supera los 3 meses y hasta los 42 meses de actividad, y emprendimiento establecido, cuando supera estos 42 meses. Esta referencia ha sido la que nos ha llevado a medir la supervivencia en $t+3$ y $t+6$, para analizar los condicionantes con mayor influencia en el estadio de nueva empresa en búsqueda de consolidación y empresa establecida, más representativa de la estabilidad a largo plazo (Kelley et al. 2011).

Los datos de estas empresas fueron extraídos de una base de datos creada, con la colaboración del Servicio de Gestión y Planificación de Programas del Instituto Valenciano de la Juventud, en el marco del Programa de Ayuda a la Creación de Empresas por Jóvenes Emprendedores. De dicha base de datos, que contaba con un total de 3.477 empresas, se extrajeron sólo aquellas con carácter social que no tuvieran valores perdidos en ninguna de las variables consideradas.

Según los criterios del IVAJ, se considera una empresa de interés social a aquella que se dedique a actividades que promuevan la igualdad de oportunidades (integración), la mejora del medio ambiente, la innovación tecnológica, la innovación organizativa, la educación y formación para la integración o la cooperación empresarial (Diario Oficial de la Comunidad Valenciana -DOCV, 2007). Todas las 
empresas participaron en el citado programa y fueron creadas por emprendedores menores de 30 años solos o en colaboración con otros mayores de esa edad (siempre que su participación fuera minoritaria). La información utilizada se ha obtenido de las memorias de proyectos presentados al programa, de las memorias anuales de ejecución del mismo y del seguimiento realizado a las empresas creadas por medio de las bases de datos de las Cámaras de Comercio de Alicante, Castellón y Valencia.

Dado que no fue posible conseguir la información sobre el momento concreto del cierre empresarial, en su caso, para medir el resultado o outcome supervivencia se han utilizado dos variables dicotómicas que indican si la empresa sobrevive o no a 31 de diciembre del tercer (caso de las empresas de reciente creación o new firms) y sexto año posterior al año de creación de la empresa (caso de las empresas consolidadas o consolidated firms). En el subapartado siguiente, se ofrece la información de las condiciones antecedentes, es decir, las variables explicativas, así como respecto a cómo fueron calibradas.

\subsection{Calibración de los datos originales}

El presente trabajo ha tomado en consideración 9 condiciones causales o condiciones antecedentes, descritas en el cuadro $n^{0} 3$. La calibración de los datos originales, de todas las condiciones y resultados, es un prerrequisito para utilizar fsQCA. Para ello, es adecuado seguir el procedimiento establecido por Ragin (2008). Todos los valores de los conjuntos difusos oscilan entre 0,00 (total no pertenencia) y 1,00 (total pertenencia). Esas puntuaciones no son probabilidades, sino un "valor verdadero". Entre esos dos puntos es necesario establecer, partiendo de la teoría y la evidencia previa, tres puntos de corte o puntos de observación para cada condición o resultado (excepto en el caso de las variables dicotómicas): 0,05 como umbral de no pertenencia plena; 0,5 como punto de indiferencia que indica que el caso no está ni dentro ni fuera, total ambigüedad; y 0,95 como umbral de pertenencia plena (Ragin, 2008).

La calibración valora el grado en que los casos cumplen o pertenecen a un patrón causal. La puntuación de pertenencia, en el caso de una configuración formada por varias condiciones causales, constituye "el grado de pertenencia a la intersección entre el conjunto difuso de condiciones causales que comprende el patrón" (Woodside y Zhang, 2013: 269). El valor de la intersección es igual a la mínima puntuación entre las condiciones a las que configuran la receta o patrón causal, utilizando los valores calibrados. En la tabla $n^{\circ} 1$ se indican los puntos de corte 0 de observación anteriormente señalados, para todas las condiciones y los resultados analizados. 


\section{Cuadro 3. Descripción de las condiciones antecedentes}

\begin{tabular}{|c|c|}
\hline CONDICIÓN ANTEDECENTE & DESCRIPCIÓN \\
\hline Plantilla inicial (plant) & $\begin{array}{l}\text { Número total de empleados estables, incluyendo emprendedores (plantilla inicial pro- } \\
\text { medio a TC). }\end{array}$ \\
\hline Capital Inicial (kin) & Proxy del capital inicial, capital subvencionable de acuerdo a los criterios del IVAJ. \\
\hline Viabilidad económica (vec)* & $\begin{array}{l}\text { Valora el análisis y situación de la competencia, las capacidades de la empresa a nivel } \\
\text { interno, los objetivos planteados y el tipo de ventajas competitivas que trata de explotar, } \\
\text { desde la lógica de la coherencia, la plantilla, el capital inicial, etc. }\end{array}$ \\
\hline Viabilidad financiera (vfin)* & $\begin{array}{l}\text { Valora las fórmulas de financiación empleadas, el nivel de endeudamiento, su capaci- } \\
\text { dad de generación de fondos y, en general, el estado de cuentas y sus proyecciones a } \\
\text { futuro. }\end{array}$ \\
\hline Viabilidad organizativa $\left(\right.$ vorg) ${ }^{\star}$ & $\begin{array}{l}\text { Valora las prácticas realizadas y previstas en relación a las distintas funciones de la } \\
\text { empresa, aprovisionamiento, producción, distribución, marketing y RRHH. }\end{array}$ \\
\hline Experiencia afín (expafin) & $\begin{array}{l}\text { Variable categórica que indica si el emprendedor tiene experiencia laboral específica } \\
\text { relacionada con el negocio. Sus valores son 1) afín (más de un año de experiencia afín); } \\
\text { 2) semiafín (más de } 3 \text { meses de experiencia afín o más de un año de experiencia semia- } \\
\text { fín); 3) no afín (no se cumplen las condiciones anteriores). }\end{array}$ \\
\hline Formación afín (formafin) & $\begin{array}{l}\text { Variable categórica que indica si el emprendedor cuenta con algún tipo de formación } \\
\text { específica relacionada con el negocio. Sus valores son 1) afín; 2) seminafín; } 3 \text { no afín. }\end{array}$ \\
\hline Nivel de formación (nivform) & $\begin{array}{l}\text { Variable categórica que recoge } 4 \text { niveles de formación: 1) estudios primarios; 2) } \\
\mathrm{ESO}+\mathrm{FPI} \text {; 3) FPII+Bachiller; 4) estudios universitarios. }\end{array}$ \\
\hline Tipo emprendedor (tipoemp) & $\begin{array}{l}\text { Variable dicotómica que señala si se trata de un emprendimiento por oportunidad (1) o } \\
\text { necesidad (0). Si el promotor (o al menos del } 75 \% \text { de los promotores si son varios) parte } \\
\text { de una situación de empleo estable por cuenta propia o ajena, consideraremos que se } \\
\text { trata de un emprendimiento por oportunidad, si no se cumple este requisito lo conside- } \\
\text { raremos emprendimiento por necesidad. }\end{array}$ \\
\hline
\end{tabular}

* Las tres variables que miden la viabilidad han sido evaluadas por expertos en el marco del programa del IVAJ partiendo de lo expuesto en el Plan de Empresa presentado por el/los promotor/es.

FUENTE: Elaboración propia. 


\section{Tabla 1. Calibración de las condiciones antecedentes y resultados/outcomes}

\begin{tabular}{|lccc|}
\hline CONDICIÓN & $\begin{array}{c}0.05 \\
\text { (No pertenencia plena) }\end{array}$ & $\begin{array}{c}0.5 \\
\text { (Punto de indiferencia) }\end{array}$ & $\begin{array}{c}0.95 \\
\text { (Pertenencia plena) }\end{array}$ \\
\hline plant & 1 & 2.1 & 4 \\
kin & 4.103 & 19.158 & 79.413 \\
vec & 0 & 35 & 70 \\
vfin & 0 & 35 & 70 \\
vorg & 0 & 35 & 70 \\
expafin & $3=0$ & $2=0.6$ & $1=1$ \\
fomafin & $3=0$ & $2=0.6$ & $1=1$ \\
nivform & $1=0$ & $3=0.75$ & $4=1$ \\
tipoemp & 0 & & 1 \\
Superv $t+3$ & 0 & & 1 \\
Superv $t+6$ & 0 & &
\end{tabular}

FUENTE: Elaboración propia.

En el apartado siguiente se presentan los resultados del análisis de necesidad y suficiencia, obtenidos al aplicar el algoritmo utilizado por el fsQCA a los datos calibrados.

\section{Resultados}

Del análisis de necesidad, hay que destacar que ninguna de las condiciones analizadas es necesaria para la supervivencia empresarial de las empresas sociales en ninguno de los dos periodos analizados (ver tabla 2). No obstante, la formación del emprendedor, la formación afín a la actividad que se realiza y la experiencia afín tienen consistencias superiores a 0.8 , lo que reafirma la importancia de la preparación previa del emprendedor, cuya importancia ya ha sido destacada en numerosos estudios (Headd, 2003; Korunka et al. 2010; Reuber y Fisher 1999; Van Praag, 2003). 


\section{Tabla 2. Análisis de necesidad para la supervivencia en $t+3$ y $t+6$}

\begin{tabular}{|c|c|c|c|c|}
\hline \multirow{2}{*}{$\begin{array}{l}\text { Resultado / Outcome } \\
\text { Condiciones analizadas }\end{array}$} & \multicolumn{2}{|c|}{ superv_t+3 } & \multicolumn{2}{|c|}{ superv_t+6 } \\
\hline & Consistencia & Cobertura & Consistencia & Cobertura \\
\hline fs_nivform & 0.887441 & 0.749000 & 0.885057 & 0.822430 \\
\hline fs_nivform & 0.112559 & 0.593750 & 0.114943 & 0.842105 \\
\hline fs_formafin & 0.868246 & 0.757025 & 0.865517 & 0.822052 \\
\hline fs_formafin & 0.131754 & 0.579167 & 0.134483 & 0.841727 \\
\hline fs_vorg & 0.665735 & 0.753110 & 0.667414 & 0.826725 \\
\hline fs_vorg & 0.334265 & 0.681581 & 0.332586 & 0.820502 \\
\hline fs_vfin & 0.606446 & 0.753282 & 0.606552 & 0.824789 \\
\hline fs_vfin & 0.393554 & 0.691251 & 0.393448 & 0.824422 \\
\hline fs_vec & 0.742464 & 0.759637 & 0.732471 & 0.813545 \\
\hline fs_vec & 0.257536 & 0.648681 & 0.267529 & 0.856643 \\
\hline fs_kin & 0.509953 & 0.807141 & 0.514138 & 0.831413 \\
\hline fs_kin & 0.490047 & 0.659902 & 0.485862 & 0.817602 \\
\hline fs_expafin & 0.802844 & 0.752889 & 0.820690 & 0.842975 \\
\hline fs_expafin & 0.197156 & 0.640000 & 0.179310 & 0.750000 \\
\hline fs_plant & 0.459242 & 0.816688 & 0.480230 & 0.862332 \\
\hline$\sim f s \_$plant & 0.540758 & 0.665889 & 0.519770 & 0.792638 \\
\hline tipoemp & 0.497630 & 0.760870 & 0.511494 & 0.847619 \\
\hline tipoemp & 0.502370 & 0.697368 & 0.488506 & 0.801887 \\
\hline
\end{tabular}

Nota: fs_ ${ }_{-}$indica presencia de la condición * $y \sim f s_{-}{ }^{*}$ indica ausencia de la misma.

FUENTE: Elaboración propia.

Por tanto, nuestra proposición 1, se cumple en sentido estricto, pues ninguna de las condiciones por sí sola es necesaria, es decir, la supervivencia de la empresa social no exige "necesariamente" la presencia 0 ausencia de un antecedente causal. Tampoco existe una combinación de estos antecedentes que resulte una condición necesaria para la supervivencia. Ahora bien, algunos de los antecedentes causales analizados, específicamente los relacionados con el background del emprendedor resultan especialmente relevantes y, por tanto, cuasi-necesarios, ya que destacan por encima del resto y sus valores de consistencia son próximos a 0.9 (Schneider et al., 2010).

Por lo que respecta al análisis de suficiencia cabe destacar que no hay un único patrón para la supervivencia de las empresas ni en $\mathrm{t}+3$ ni en $\mathrm{t}+6$. Concretamente, en ambos casos, existen 4 caminos o recetas que, en general, conducen a la supervivencia de las empresas sociales, si bien, como veremos más adelante, varían parcialmente de un periodo a otro. Si nos centramos en la supervivencia de empresas de nueva creación, en $\mathrm{t}+3$, (ver tabla 3), las 4 soluciones explican un $29 \%$ de 
los casos, lo que, sin ser un porcentaje muy elevado, no resulta desdeñable y es similar a los niveles de cobertura de estudios precedentes. El nivel de consistencia es elevado y alcanza un valor algo superior a 0,88 . La cobertura bruta de los distintos patrones causales oscila entre 0,12 y 0,18 , siendo la cobertura neta de las soluciones 2 y 4 bastante superior a la de las demás.

\section{Tabla 3. Solución de mayor parsimonia para la supervivencia en $t+3$}

\begin{tabular}{|c|c|c|c|c|c|c|c|c|c|c|c|c|}
\hline \multirow[b]{2}{*}{ 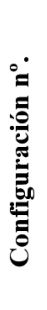 } & \multirow[b]{2}{*}{ 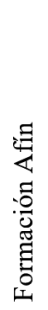 } & \multirow[b]{2}{*}{ 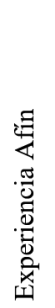 } & \multicolumn{5}{|c|}{ Condiciones antecedentes } & \multirow[b]{2}{*}{ 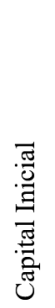 } & \multirow[b]{2}{*}{ 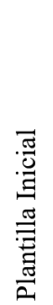 } & \multicolumn{2}{|c|}{ Cobertura } & \multirow[t]{2}{*}{ Consistencia } \\
\hline & & & 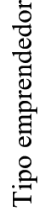 & 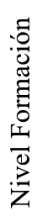 & 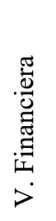 & 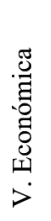 & 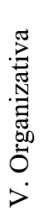 & & & Bruta & $\begin{array}{c}\text { Neta o } \\
\text { específica }\end{array}$ & \\
\hline 1 & 0 & 0 & & 0 & 0 & 0 & 0 & 0 & & 0.117014 & 0.026019 & 0.847289 \\
\hline 2 & 0 & 0 & & ○ & & 0 & 0 & 0 & 0 & 0.178483 & 0.046730 & 0.891361 \\
\hline 3 & 0 & - & & ○ & & 0 & 0 & 0 & & 0.137488 & 0.021896 & 0.899535 \\
\hline 4 & 0 & & 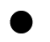 & 0 & 0 & 0 & 0 & 0 & 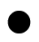 & 0.133175 & 0.044929 & 0.857492 \\
\hline
\end{tabular}

Cobertura de la solución: 0.288199

Consistencia de la solución: 0.880284

Nota: Los círculos negros indican la presencia de una condición antecedente. Los círculos blancos indican la ausencia o negación de una condición antecedente. Las celdas en blanco representan condiciones ambiguas. Umbral de Frecuencia $=5$; Umbral de Consistencia $=.80$.

FUENTE: Elaboración propia.

Destacan por su relevancia cinco de las condiciones analizadas, que aparecen en todas las configuraciones que llevan a la supervivencia en $t+3$. Estas son: formación, formación afín, viabilidad económica, viabilidad organizativa y el capital inicial. Sorprendentemente, si bien la literatura destaca las dificultades de las empresas sociales para conseguir recursos financieros, la viabilidad financiera del plan presentado no aparece como un elemento relevante a la hora de explicar la supervivencia de la empresa social en los tres primeros años de vida.

Por lo que respecta a la experiencia afín, presente en 3 de las 4 configuraciones, parece jugar un papel relevante a corto plazo, si bien no imprescindible. Un emprendedor social menos "experto" tiene considerables probabilidades de sobrevivir durante los primeros años si cuenta con mejores recursos financieros y una plantilla más amplia y una motivación poderosa para llevar adelante la iniciativa. A este respecto cabe recordar que el emprendedor por oportunidad tiene una mayor ten- 


\section{SUPERVIVENCIA DE EMPRESAS SOCIALES DE NUEVA CREACIÓN. UN ENFOQUE BASADO EN EL ANÁLISIS CUALITATIVO COMPARATIVO FSQCA}

dencia a perseverar en el intento y suele ser más innovador. Por otro lado, otros miembros de la empresa podrían quizás suplir las carencias del emprendedor o complementar sus conocimientos, mientras que la solidez financiera podría permitirle absorber mejor los costes del "prueba y error".

Como señalábamos anteriormente, la ausencia de viabilidad financiera no parece penalizar la supervivencia de las empresas de reciente creación en el caso de emprendedores con formación y experiencia, que presentan un proyecto con alta viabilidad económica y organizativa y elevado capital inicial, sino que la favorece. Quizás sea el propio carácter social que lleva a algunas de estas empresas a "fiar", en cierto modo, su supervivencia a aportaciones en forma de subvenciones y donaciones cuando la viabilidad financiera mide, principalmente, su capacidad de autofinanciación. Por otro lado, el contar con un capital inicial por encima de la media, facilita, al menos a corto plazo su supervivencia. Por otro lado, quizás esa carencia de la empresa, lleva al emprendedor a "hacer de la necesidad virtud" y buscar soluciones creativas aprovechando y recombinando esos recursos escasos, convirtiéndose en un bricoleur (Baker y Nelson, 2005; Senyard et al. 2014). No obstante, los resultados obtenidos en $t+6$ parecen desmentir, al menos parcialmente, este supuesto.

En todo caso, a corto plazo parece especialmente relevante contar con unos mínimos recursos financieros iniciales, aunque la forma de financiar la inversión sea incierta o de alto riesgo (Por ejemplo, mediante la hipoteca de los bienes inmuebles propiedad del emprendedor o préstamos familiares, prácticas muy comunes entre las empresas analizadas), y no se tenga un plan financiero adecuadamente elaborado, lo que llevaría a una evaluación negativa por parte de los expertos.

Al margen de la viabilidad financiera, los otros dos condicionantes que menos aparecen son el tipo de emprendedor, según la motivación para emprender, y la plantilla, que aparecen en solo 2 de las 4 configuraciones. Por lo que respecta a la distinción emprendedor por oportunidad/necesidad, a priori, la misma parece tener menos sentido en el caso de empresas sociales que en el de las empresas de negocio. No obstante, los emprendedores las pueden ver como una doble vía de contribuir a la sociedad y al bienestar propio. Además, cabe significar que la mayoría de las empresas de la muestra no se pueden considerar puramente sociales sino híbridas, en el sentido de que persiguen simultáneamente objetivos sociales y económicos (Massetti, 2008). Otra razón por la cual este condicionante puede no aparecer como especialmente determinante puede derivar de lo expuesto por autores como Giacomin et al. (2011), cuando señalan que ambos tipos de motivación pueden coexistir.

Por último, el contar con una plantilla mayor, sólo constituye un condicionante relevante en dos de las cuatro configuraciones. Al margen de lo ya comentado al indicar este condicionante como "parcialmente compensatorio" de la falta de experiencia del emprendedor, cabe considerar que puede jugar un papel más relevante a más largo plazo. En este sentido Revuelto-Taboada y Simón-Moya (2015) plantean que el capital inicial tendría una influencia superior que el capital humano en la supervivencia a corto plazo, adquiriendo este una mayor relevancia en el largo plazo. No obstante, nuestros resultados lo desmienten a la luz de lo que presentamos a continuación. 
Al centrar la atención en la supervivencia de las empresas sociales en $t+6$, empresas que consideramos ya consolidadas conforme a los criterios de GEM (ver tabla 4), se repite en términos generales el patrón, si bien cambia alguna de las variables más relevantes. De nuevo, cinco variables están presentes en las cuatro combinaciones que parecen llevar a la supervivencia. Estas variables son la formación, la formación afín, la experiencia afín, la viabilidad económica y la viabilidad organizativa. En este caso, el ratio de cobertura global es mayor que en el caso anterior, pues supera el $34 \%$, con un nivel de consistencia que supera el $83 \%$. La cobertura bruta de los distintos patrones causales oscila entre 0,14 y 0,23 , presentando una menor diferencia en cuanto a cobertura neta respecto a $t+3$.

\section{Tabla 4. Solución de mayor parsimonia para la supervivencia en $t+6$}

Condiciones antecedentes

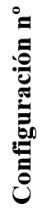

ن

1

2

3

4
Cobertura

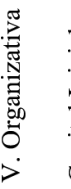

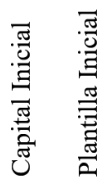

Bruta

Neta o específica

Cobertura de la solución: 0.342529

Consistencia de la solución: 0.832519

Nota: Los círculos negros indican la presencia de una condición antecedente. Los círculos blancos indican la ausencia o negación de una condición antecedente. Las celdas en blanco representan condiciones ambiguas. Umbral de Frecuencia = 5; Umbral de Consistencia $=.80$.

FUENTE: Elaboración propia.

Como diferencias destacables respecto a la supervivencia en $t+3$, la variable capital inicial deja de estar presente en todas las configuraciones causales, mientras se mantienen las demás, y se añade la experiencia afín. Es decir, al igual que en $t+3$ la viabilidad económica y organizativa del proyecto de empresa y el background del emprendedor social, donde la experiencia adquiere un rol todavía más determinante, resultando de especial relevancia para que la empresa social se consolide. Por lo que respecta a la motivación para emprender o tipo de emprendedor, la situación no varía entre $t+3$ y $t+6$, por lo que siguen teniendo un papel secundario

También cabe destacar que en $t+6$, a diferencia del periodo $t+3$, la viabilidad financiera también se muestra como una condición bastante relevante para la supervivencia empresarial en las empre- 
sas sociales, apareciendo en 3 de las 4 configuraciones causales establecidas. Sólo desaparece en la cuarta solución, en la que adquiere relevancia la motivación por oportunidad. Como era de esperar, el papel del capital inicial pasa, a lo largo del tiempo, a un rol secundario, mientras el equilibrio financiero y la capacidad de la empresa para financiarse adecuadamente, que es lo que valora la viabilidad financiera, adquieren más importancia.

En definitiva, por lo que respecta a las proposiciones 2 y 3 , cabe destacar que la existencia de 4 patrones causales diferentes, que combinan diversas condiciones antecedentes todos ellos, tanto para explicar la supervivencia en t+3 como en t+6, constituye una evidencia a favor de la proposición 2. Es decir, distintas combinaciones de antecedentes causales pueden llevar a la supervivencia. Ello supone aceptar la existencia de cierta "equifinalidad", pues algunas carencias en determinadas características pueden "salvarse" por medio de otros recursos o fortalezas.

Por otro lado, si bien existen similitudes entre los patrones que explican la supervivencia de las empresas de nueva creación y las consolidadas, también existen algunas diferencias entre los mismos, como se planteaba en la proposición 3. Lo más destacable es el cambio de "pesos" relativos a la hora de explicar la supervivencia entre el capital inicial y la viabilidad financiera. Ello nos lleva a pensar que en una primera etapa es más el capital inicial que la capacidad de bricolaje, la que sostiene financieramente a las empresas.

Todo lo anterior nos indica que no existe una única forma de alcanzar el resultado, en este caso, conseguir sobrevivir, y que la importancia relativa de los distintos condicionantes puede variar a lo largo del tiempo, conforme la empresa va adquiriendo una mayor madurez.

\section{Conclusiones y futuras líneas de investigación}

Los resultados obtenidos explican un porcentaje significativo, aunque minoritario, de casos de empresas supervivientes. Revelan la importancia de la formación y experiencia del emprendedor, así como de la viabilidad económica y organizativa del proyecto de empresa. El tamaño de la plantilla y el tipo de motivación no parecen jugar un papel relevante. No obstante, cabe señalar a este respecto, en primer lugar, que la dispersión en cuanto a tamaño de las empresas de la muestra no es grande y que la media de empleados por empresa es inferior a dos al tratarse, en la gran mayoría de los casos, de fenómenos de autoempleo o microempresas. Por otro lado, como ya comentamos anteriormente, la motivación para emprender puede ser mixta y quizás pierde, en cierto modo, su sentido al tratarse de empresas sociales en las cuales resulta relevante lo que autores como Grant (2008) y Penner et al. (2005) denominan motivación prosocial, es decir, al deseo de beneficiar a otras personas, incluso en ausencia de contraprestaciones pecuniarias. Ello supone una motivación altruista, más 
relacionada con el descubrimiento de una oportunidad para dar cobertura a un problema social, si bien tampoco excluye la posibilidad de convertir la actividad en una carrera profesional e incluso hacer de ello un medio de vida.

No se detectan grandes diferencias, en cuanto a cuáles son los factores relacionados con la supervivencia de las empresas sociales, entre las empresas de reciente creación y las consolidadas. No obstante, el capital inicial, que juega un papel importante en la primera etapa de la vida de la empresa, pierde relevancia conforme esta se consolida, mientras su capacidad para obtener recursos y dotarse de una estructura financiera sólida adquiere mayor relevancia. En todo caso, la existencia de diversas soluciones suficientes que, además, varían ligeramente según el periodo considerado, nos lleva a concluir que no existe una única forma de conseguir sobrevivir, y que la importancia relativa de los distintos condicionantes puede variar conforme la empresa va adquiriendo una mayor madurez. También indica que la ausencia de una característica determinada puede, en cierto modo, "compensarse" con la presencia de otras.

Los resultados obtenidos no muestran diferencias sustanciales a los recogidos en trabajos centrados en analizar la supervivencia de empresas comerciales o de negocio, que han utilizado variables o condicionantes similares. No obstante, estos estudios, han sido realizados casi sin excepción utilizando técnicas estadísticas tradicionales, por lo que revelan muy poco en relación a las posibles interacciones entre las variables explicativas o condicionantes (Simón-Moya y Revuelto-Taboada. 2016). En todo caso, el background del emprendedor aparece en ambos casos como relevante, así como el capital inicial, especialmente en empresas jóvenes. Como diferencias más destacables, la mayor relevancia de la viabilidad del proyecto empresarial (en especial de su viabilidad organizativa y económica), y la ya comentada menor capacidad explicativa de la motivación para emprender.

Lo más destacable de los resultados obtenidos utilizando el Análisis Cualitativo Comparativo, es que nos permite observar el efecto conjunto de distintas combinaciones de antecedentes causales. Más concretamente, de la interpretación de los resultados se desprende, por ejemplo, que un buen proyecto, incluso cuando es impulsado por un emprendedor con formación y experiencia, puede fracasar a corto plazo si no cuenta con un mínimo de capital. Ahora bien, la viabilidad futura del proyecto dependerá más que de este capital inicial de su capacidad para obtener recursos y dotarse de una estructura financiera sólida, lo que está más directamente relacionado con su capacidad de gestión y la legitimidad y viabilidad alcanzada por la empresa social.

El presente trabajo ofrece ciertas contribuciones prácticas, en la medida en que puede ofrecer ciertas guías a los responsables de políticas públicas orientadas a la promoción del emprendimiento. En primer lugar, nos lleva a insistir en la necesidad de potenciar la formación, especialmente cuando es de orden teórico-práctico y afín a la actividad que se pretende emprender. Por otro lado, a la hora de evaluar solicitudes de cara a la concesión de ayudas, parece recomendable tener muy en cuenta, además de su carácter social, que puede generar beneficios sociales adicionales al empleo y riqueza creados por la empresa, variables objetivas y fáciles de evaluar como la formación y experiencia afín 


\section{SUPERVIVENCIA DE EMPRESAS SOCIALES DE NUEVA CREACIÓN. UN ENFOQUE BASADO EN EL ANÁLISIS CUALITATIVO COMPARATIVO FSQCA}

y el capital inicial que aporta el emprendedor, sin los cuales la viabilidad del proyecto (algo que resulta mucho más difícil de evaluar) para nada garantiza la supervivencia.

Otro tipo de programas de promoción de la creación de empresas sociales, centrados en el asesoramiento previo y el seguimiento posterior a la constitución de las mismas, podrían hacer una contribución importante al emprendimiento y a la sociedad, tomando como eje central el plan de empresa. Estos programas, que podrían estar o no vinculados a la obtención de ayudas financieras, pueden resultar muy eficaces a la hora de aumentar las posibilidades de éxito de las empresas de reciente creación, por la vía de mejorar su proceso de formulación e implementación del plan de empresa, y completar la formación inicial de los emprendedores supliendo inicialmente algunas de sus carencias en materia de gestión. Cabe recordar que la falta de apoyo y asesoramiento externo se ha señalado en la literatura como una de las carencias de las empresas sociales. Otras formas de suplir estas carencias podrían derivar del asociacionismo y cooperación interempresarial, con el apoyo de instituciones públicas y privadas de las que ya existen ejemplos comentados al inicio de este trabajo.

Por lo que respecta a las limitaciones del presente trabajo cabe significar que el estudio ha sido desarrollado en el ámbito de la Comunidad Autónoma Valenciana, con una muestra de emprendedores menores de 30 años o menores de 30 años acompañados de emprendedores de mayor edad. Todo ello limita la posibilidad de generalizar los resultados, por lo que resultaría conveniente en posteriores investigaciones replicar el estudio en un ámbito geográfico diferente. Asimismo, convendría utilizar una muestra que abarcase emprendedores de diferentes edades, lo que probablemente haría que la experiencia previa jugase un papel más determinante a la hora de explicar las probabilidades de supervivencia de las empresas.

Por otro lado, resulta difícil establecer si los planes de empresa se elaboran, en gran medida, con el objetivo de conseguir ayudas públicas, sin que existan, en muchos casos, ni las capacidades necesarias para implementarlos, ni la verdadera intención de hacerlos servir de guía para el desarrollo del proyecto. A este respecto, podría plantearse, al menos inicialmente, un estudio de casos que permitiese analizar las desviaciones existentes entre lo que inicialmente se proponía y lo realizado.

Obviamente, otra limitación que seguramente ha reducido la capacidad explicativa del modelo, deriva de no haber considerado otras variables potencialmente relevantes, como pueden ser: la fase del ciclo económico en que inician su actividad las empresas analizadas; las peculiaridades de su sector de actividad; la estrategia de la empresa u otras como el género y las características psicológicas del emprendedor. Variables que deberían ser tenidas en cuenta en estudios posteriores. 


\section{Bibliografía}

ACS, Z.J., BOARDMAN, M.C. \& MCNEELY, C.L. (2013): "The social value of productive entrepreneurship", Small Business Economics, 40(3): 785-796.

ALVORD, S.H., BROWN, L.D. \& LETTS, C.W. (2004): "Social entrepreneurship and societal transformation, an exploratory study", The Journal of Applied Behavioral Science, 40(3): 260-282.

AMABILE, T.M., CONTI, R., COON, H., LAZENBY, J. \& HERRON, M. (1996): "Assessing the work environment for creativity", Academy of Management Journal, 39(5): 1154-1184.

ARTHUR, S., KEENOY, T., SCOTT-CATO, M. \& SMITH, R. (2006): "Where is the social in social enterprise?", Alternative spaces of economy, society and politics: interrogating alterity, D. Fuller, A.E.G. Jonas y R. Lee (Eds.), Aldershot, UK: Ashgate: 207-222.

AUSTIN, J., STEVENSON, H. \& WEI-SKILLERN, J. (2006): "Social and Commercial Entrepreneurship: Same, Different, or Both?", Entrepreneurship Theory and Practice, 30(1): 1-22.

BAGLIONI, S. (2017): "A Remedy for All Sins? Introducing a Special Issue on Social Enterprises and Welfare Regimes in Europe", VOLUNTAS: International Journal of Voluntary and Nonprofit Organizations, 28(6): 2325-2338.

BAKER, T. \& NELSON, R.E. (2005): "Creating something from nothing: Resource construction through entrepreneurial bricolage", Administrative Science Quarterly, 50(3): 329-366.

BEUGRÉ, C.D. (2011): USASBE. Disponible en: http://sbaer.uca.edu/research/USASBE/2011/ PaperID61.pdf; consultado 24/09/2013.

BORNSTEIN, D. \& DAVIS, S. (2010): Social Entrepreneurship. What everyone needs to know, Oxford University Press, Oxford, UK.

BOSCHEE, J. (1998): Merging Mission and Money: A board member's guide to social entrepreneurship, National Center for non-profit boards.

BOSMA, N. \& LEVIE, J. (2009): 2009 Global Report del Global Entrepreneurship Monitor, de http://www.gemconsortium.org/docs/ download/265; Recuperado el 15/06/2011.

BRINCKERHOFF, P.C. (2000): Social Entrepreneurship: The art of Mission-Based Venture Development, John Wiley \& Son, Nueva York.

BROUARD, F. \& LARIVET, S. (2010): Essay of clarifications and definitions of the related concepts of social enterprise, social entrepreneur and social entrepreneurship, Handbook of research on social entrepreneurship: 29-56. 
BRÜDERL, J., PREISENDÖRFER, P. \& ZIEGLER, R. (1992): "Survival chances of newly founded business organizations", American Sociological Review, 57(2): 227-242.

BRÜDERL, J. \& SCHÜSSLER, R. (1990): "Organizational mortality: The liabilities of newness and adolescence", Administrative Science Quarterly, 35(3): 530-537.

BUSSTRA, T.J. \& VERHOEF, L.H.J. (1993): Starten op z'n best; slaagen faalfactoren van jonge ondernemingen, Amsterdamse Academie voor Bank en Financiën, Amsterdam.

CANTARERO, S., GONZÁLEZ-LOUREIRO, M. \& PUIG, F. (2017): "Efectos de la crisis económica sobre el emprendimiento en empresas de economía social en España: un análisis espacial", REVESCO, Revista de Estudios Cooperativos, 125: 24-48.

CASTROGIOVANNI, G.J. (1996): "Pre-startup planning and the survival of new small businesses: Theoretical linkages", Journal of Management, 22(6): 801-822.

CESE (2011): Dictamen del Comité Económico y Social Europeo sobre «Espíritu empresarial social y las empresas sociales", (Dictamen exploratorio) (2012/C 24/01), Diario Oficial de la Unión Europea 28///2012.

CHANDRA, Y. \& SHANG, L. (2017): "Unpacking the biographical antecedents of the emergence of social enterprises: A narrative perspective", VOLUNTAS: International Journal of Voluntary and Nonprofit Organizations, 28(6): 2498-2529.

CHANDRA, Y. \& WONG, L. (Eds.) (2016): Social Entrepreneurship in the Greater China Region: Policy and Cases, Vol. 147, Routledge.

CHAPMAN, T., FORBES, D. \& BROWN, J. (2007): "They have God on their side: the impact of public sector attitudes on the development of social enterprise", Social Enterprise Journal, 3(1): 78-89.

CIRUELA, A.M., CUADRADO, M. \& PLAZA, J.J. (2016): "La economía social como alternativa de autoempleo. El perfil del emprendedor/a en las sociedades laborales andaluzas", REVESCO, Revista de Estudios Cooperativos, 122: 59-85.

CONWAY, C. (2008): "Business planning training for social enterprise", Social Enterprise Journal, 4(1): 57-73.

COOPER, A.C., GIMENO, F.J. \& WOO, C.Y. (1994): "Initial human and financial capital as predictors of new venture performance", Journal of Business Venturing, 9(5): 371-395.

CURTO, M. (2012): "Los emprendedores sociales: innovación al servicio del cambio", Cuadernos de la Cátedra 'La Caixa' de Responsabilidad Social y Gobierno Corporativo, 13: 1-23.

DEES, J.G. (2001): The meaning of social entrepreneurship, http://web.mit.edu/sloan2/dese/ readings/week01/Dees_TheMeaningofSocialEntrepreneurship.pdf 
DEFOURNY, J. \& NYSSENS, M. (2012): "El enfoque EMES de la empresa social desde una perspectiva comparada", CIRIEC-España, Revista de Economía Pública, Social y Cooperativa, 75, 634.

DEFOURNY, J. \& NYSSENS, M. (2017): "Fundamentals for an international typology of social enterprise models", VOLUNTAS: International Journal of Voluntary and Non-Profit Organizations, 28(6): 2469-2497.

DELMAR, F. \& SHANE, S. (2003): "Does business planning facilitate the development of new ventures?", Strategic Management Journal, 24(12): 1165-1185.

DENNY, S. (2014): Who lives the longest? Busting the social venture survival mith, E3M.

DI DOMENICO, M., HAUGH, H. \& TRACEY, P. (2010): "Social Bricolage: Theorizing Social Value Creation in Social Enterprises", Entrepreneurship Theory and Practice, 34(4): 681-703.

DORADO, S. (2006): "Social Entrepreneurial ventures: different values so different process of creation, no?", Journal of Development Entrepreneurship, 11(4): 1-24.

DRAYTON, W. (2002): "The Citizen Sector: Becoming as Entrepreneurial and Competitive as Business", California Management Review, 44(3): 120-133.

EL HARBI, S. \& ANDERSON, A.R. (2010): "Institutions and the Shaping of Different Forms of Entrepreneurship", The Journal of Socio-Economics, 39(3): 436-444.

ENCISO, M., GÓMEZ, L. \& MUGARRA, A. (2012): "La iniciativa comunitaria en favor del emprendimiento social y su vinculación con la economía social: una aproximación a su delimitación conceptual", CIRIEC-España, Revista de Economía Pública, Social y Cooperativa, 75, 55-80.

FERNÁNDEZ-GUERRERO, R. \& REVUELTO-TABOADA, L. (2009): Informe sobre el emprendimiento de jóvenes en la Comunidad Valenciana 2000-2008, Documento de trabajo no publicado, UVEG - IVAJ.

FEURER, S., BAUMBACH, E. \& WOODSIDE, A.G. (2015): "Applying configurational theory to build a typology of ethnocentric consumers", International Marketing Review, 33(3): 351-375.

FISS, P.C. (2011): "Building better causal theories: A fuzzy set approach to typologies in organization research", Academy of Management Journal, 54(2): 393-420.

FOURNIER, A.N. (2011): Emprendimiento Social. Documento de investigación. Administración de Empresas, $n^{\circ} 6$. Ediciones Fundación Universidad Central: 46.

FRANCO, M. \& HAASE, H. (2010): "Failure factors in small and medium-sized enterprises: Qualitative study from an attributional perspective", International Entrepreneurship and Management Journal, 6(4): 503-521.

GARUD, R. \& KARNOE, P. (2003): "Bricolage versus breakthrough: Distributed and embedded agency in technology entrepreneurship", Research Policy, 32: 277-300. 
GEM (2009): Global Entrepreneurship Monitor. 2009 Global Report, Instituto de Empresa, Madrid, España.

GIACOMIN, O., JANSSEN, F., GUYOT, J.L. \& LOHES, L. (2011): Opportunity and/or necessity entrepreneurship? The impact of the socio-economic characteristics of entrepreneurs, http://mpra.ub.unimuenchen.de/29506/, recuperado el 05/04/2015.

GIMMON, E. \& SPIRO, S. (2013): "Social and commercial ventures: A comparative analysis of sustainability", Journal of Social Entrepreneurship, 4(2): 182-197.

GOODSON, P., SMITH, M.M., EVANS, A., MEYER, B. \& GOTTLIEB; N.H. (2001): "Maintaining Prevention in Practice: Survival of PPIP in Primary Care Settings", American Journal of Preventive Medicine, 20(3): 184-189.

GRANT, A.M. (2008): "Does intrinsic motivation fuel the prosocial fire? Motivational synergy in predicting persistence, performance and productivity", Journal of Applied Psychology, 93(1): 48-58.

HABER, S. \& REICHEL, A. (2005): "Identifying performance measures of small ventures - the case of the tourism industry", Journal of Small Business Management, 43(3): 257-286.

HARDING, R. (2004): "Social Enterprise: The New Economic Engine", Business Strategy Review, 15(4): 39-43.

HAUGH, H. (2007): "Community-Led Social Venture Creation", Entrepreneurship Theory and Practice, 31(2): 161-182.

HEADD, B. (2003): "Redefining business success: Distinguishing between closure and failure", Small Business Economics, 21(1): 51-61.

HERNÁNDEZ, J., TIRADO, P. \& ARIZA, A. (2016): "El concepto de innovación social: ámbitos, definiciones y alcances teóricos", CIRIEC-España, Revista de Economía Pública, Social y Cooperativa, 88: 165-199.

HO, Y. \& WONG, P. (2007): "Financing, regulatory costs and entrepreneurial propensity", Small Business Economics, 28(2): 187-204.

HONIG, B. \& KARLSSON, T. (2004): "Institutional forces and the written business plan", Journal of Management, 30(1): 29-48.

KACHLAMI, H. (2016): "Social venture creation and the influence of commercial ventures", Social Enterprise Journal, 12(3): 347-367.

KATO, S., ASHLEY, S.R., \& WEAVER, R.L. (2017): "Insights for Measuring Social Value: Classification of Measures Related to the Capabilities Approach", VOLUNTAS: International Journal of Voluntary and Nonprofit Organizations, 1-24.

KELLEY, D.J., SINGER, S. \& HERRINGTON, M. (2011): 2011 Global Report. Global Entrepreneurship Monitor, http://www.gemconsortium.org/docs/2409/gem-2011-globalreport; recuperado 03/03/2013. 
KERR, W. \& NANDA, R. (2011): "Financing constraints and Entrepreneurship". En Audretsch, D., Falck, O. and Heblich, S. (Eds.), Handbook on research on Innovation and Entrepreneurship, Chentelham: Edward Elgar Publishing Limited: 88-103.

KIRZNER, I.M. (1979): Perception, Opportunity, and Porfit. Chicago: The University of Chicago Press.

KORUNKA, C., KESSLER, A., FRANK, H. \& LUEGER, M. (2010): "Personal characteristics, resources, and environment as predictors of business survival", Journal of Occupational and Organizational Psychology, 83(4): 1025-1051.

LEPOUTRE, J., JUSTO, R., TERJENSEN, S. \& BOSMA, N. (2011): "Designing a global standardized methodology for measuring social entrepreneurship activity: the Global Entrepreneurship Monitor social entrepreneurship study", Small Business Economics, 40(3): 693-714.

LIGHT, C.L. (2006): "Reshaping social entrepreneurship", Stanford Social Innovation Review, 4(3): 4551.

LÓPEZ-GARCÍA, P. \& PUENTE, S. (2006): "Business demography in Spain: determinants of firm survival", Banco de España Research Paper, No. WP-0608.

LUK, S. (1996): "Success in Hong Kong: factors self-reported by successful small business owners", Journal of Small Business Management, 34(3): 68-75.

LUSSIER, R.N. \& HALABI, C.E. (2010): "A three-country comparison of the business success versus failure prediction model", Journal of Small Business Management, 48(3): 360-377.

MASA, C.I., ITURRIOZ, J. \& MARTÍN, S. (2016): "Aspectos determinantes del fracaso empresarial: efecto de la proyección social de las sociedades cooperativas frente a otras formas jurídicas", CIRIEC-España, Revista de Economía Pública, Social y Cooperativa, 88, 93-125.

MASTíN, S., BELLOSTAS, A.J. \& LÓPEZ, F.J.L. (2017): “¿Son los voluntarios un recurso sustitutivo de los trabajadores? Una aproximación desde la economía social aragonesa", REVESCO, Revista de Estudios Cooperativos, 123, 143-173.

MASSETTI, B.L. (2008): "The Social Entrepreneurship Matrix as a "Tipping Point" for Economic Change", Emergence: Complexity and Organization, 3(10): 1-8.

MEYSKENS, M., ROBB POST, C., STAMP, J.A., CARSRUD, A.L. \& REYNOLDS, P.D. (2010): "Social Ventures From a Resource Based Perspective: An Exploratory Study Assessing Global Ashoka Fellows", Entrepreneurship Theory and Practice, 34(4): 661-80.

MIZIC, N. \& JACOBSON, R. (2003): "Trading off between value creation and value appropriation: The financial implications of shifts in strategic emphasis", Journal of Marketing, 67(1): 63-76.

MONZÓN, J.L. (2016): "La economía social en la literatura económica y en los hechos. 30 años del CIRIEC-España", CIRIEC-España, Revista de Economía Pública, Social y Cooperativa, 88: 287307. 
NAVAJAS-ROMERO, V., LÓPEZ-MARTíN, M.C. \& ARIZA-MONTES, A. (2017): "Los trabajadores autónomos dependientes en Europa", CIRIEC-España, Revista de Economía Pública, Social y Cooperativa, 89, 167-198.

NICOLÁS, C. \& RUBIO, A.M. (2012): "El emprendimiento social: una comparativa entre España y países sudamericanos", FAEDYPE International Review, 1(1): 38-49.

ORSER, B.J., HOGARTH-SCOTT, S. \& RIDING, A. (2000): "Performance, firm size and management problem solving", Journal of Small Business Management, 38(4): 42-58.

PEARCE, J. (2003): Social Enterprise in Any Town, Calouste Gulbenkian Foundation, London.

PENNER, L.A., DOVIDIO, J.F., PILIAVIN, J.A. \& SCHROEDER, D.A. (2005): "Prosocial behavior: Multilevel perspectives", Annual Review of Psychology, 56(14): 1-28.

PEREDO, A.M. \& MCLEAN, M. (2006): "Social Entrepreneurship: A Critical Review of the Concept", Journal of World Business, 41(1): 56-65.

PERAZA, E.H., GÓMEZ, J.M. \& ALEIXANDRE, G. (2016): "Los factores determinantes del comportamiento innovador de las cooperativas: un análisis para el caso de Castilla y León", REVESCO, Revista de Estudios Cooperativos, 122: 252-284.

PERRY, S.C. (2001): "The relationship between written business plans and the failure of small businesses in the U.S.", Journal of Small Business Management, 39(3): 201-208.

PICCIOTTI, A. (2017): "Towards sustainability: The innovation paths of social enterprise", Annals of Public and Cooperative Economics, 88(2): 233-256.

RAGIN, C.C. (2008): "Measurement versus calibration: A set-theoretic approach". In J.J. BoxSteffensmeier, H. Brady, y D. Collier (Eds.), The Oxford handbook of political methodology, Oxford, UK: Oxford University Press: 174-198.

RAGIN, C.C. \& DAVEY, S. (2014): Fuzzy-Set/Qualitative Comparative Analysis 2.5, Irvine, California: Department of Sociology, University of California.

RAGIN, C.C. \& FISS, P.C. (2008): "Net effects versus configurations: An empirical demonstration". In C.C. Ragin (Ed.), Redesigning social inquiry: Fuzzy sets and beyond, Chicago, IL: University of Chicago Press: 190-212.

REID, G.C. \& SMITH, J.A. (2000): "What makes a new business start-up successful?", Small Business Economics, 14(3): 165-182.

REUBER, A.R. \& FISCHER, E. (1999): "Understanding the consequences of founders' experience", Journal of Small Business Management, 37(2): 30-45.

REVUELTO-TABOADA, L. \& SIMÓN-MOYA, V. (2015): "Influence of human and physical capital on the survival of new ventures", Perspectiva Empresarial, 2(1): 45-59. 
RIBEIRO-SORIANO, D. \& CASTROGIOVANNI, G. (2012): "The impact of CEO education, experience, and inner circle advisors on SME performance: Insights from a study in public development centers", Small Business Economics, 38(3): 333-349.

ROBERTS, D. \& WOODS, C. (2005): "Changing the world on a shoestring: The concept of social entrepreneurship", University of Auckland Business Review, 7(1): 45-51.

SANDBERG, W.R., ROBINSON, R. \& PEARCE, J.A. Jr. (2001): "One more time... Should small companies attempt strategic planning?", Business and Economic Review, 48(1): 12-15.

SANTOS, F.M. (2012): "A positive theory of social entrepreneurship", Journal of business ethics, 111(3): 335-351.

SAPAG, N. \& SAPAG, R. (2003): Preparación y evaluación de proyectos, 4ª edición, México: McGrawHill.

SCHNEIDER, M.R., SCHULZE-BENTROP, C. \& PAUNESCU, M. (2010): "Mapping the institutional capital of high-tech firms: A fuzzy-set analysis of capitalist variety and export performance", Journal of International Business Studies, 41(2): 246-266.

SCHUTJENS, V.A.J.M. \& WEVER, E. (2000): "Determinants of new firm success", Papers in Regional Science, 79(2): 135-159.

SCOTT, J.A. (2001): After the bubble: investing in internet-based social enterprises in challenging times, Report for Flatiron Foundation and the Atlantic Philanthropies.

SENYARD, J., BAKER, T., STEFFENS, P. \& DAVIDSSON, P. (2014): "Bricolage as a Path to Innovativeness for Resource-Constrained New Firms", Journal of Product Innovation Management, 31(2): 211-230.

SHANE, S. \& VENKATARAMAN, S. (2000): "The promise of entrepreneurship as a field of research", Academy of Management Review, 25(1): 217-226.

SHARIR, M. \& LERNER, M. (2006): "Gauging the success of social ventures initiated by individual social entrepreneurs", Journal of world business, 41(1): 6-20.

SIMÓN-MOYA, V. \& REVUELTO-TABOADA, L. (2016): "Revising the predictive capability of business plan quality for new firm survival using qualitative comparative analysis", Journal of Business Research, 69(4): 1351-1356.

SIMÓN-MOYA, V., REVUELTO-TABOADA, L. \& RIBEIRO-SORIANO, D. (2012): "Are success and survival factors the same for social and business ventures?", Service Business, 6(2): 219-242.

SIMÓN-MOYA, V., SASTRE-SÁNCHEZ, O. \& REVUELTO-TABOADA, L. (2015): "El emprendedor social: análisis de la alerta social", Suma de Negocios, 6(14): 155-165.

STARR, J.A. \& MACMILLAN, I.C. (1990): "Resource cooptation via social contracting: Resource acquisition strategies for new ventures", Strategic Management Journal, 11: 79-92. 
STEADMAN, H.J., COCOZZA, J.J., DENNIS, D.L., LASSITER, M.G., RANDOLPH, F.L., STEWART, K.S. (2002): "Formal business planning and small business success: a survey of small business with an international focus", Journal of American Academy of Business, 2(1): 42-46.

STINCHCOMBE, A.L. (1965): "Social structures and organizations". In J.G. March (Ed.), Handbook of organizations, Chicago: Rand McNally: 142-193.

SZYMAŃSKA, A. \& JEGERS, M. (2016): "Modelling Social Enterprises", Annals of Public and Cooperative Economics, 87(4): 501-527.

THOMPSON, J.L. (2002): "The world of the social entrepreneur", The International Journal of Public Sector Management, 15(5): 412-431.

VAN PRAAG, C.M. (2003): "Business survival and success of young small business owners", Small Business Economics, 21(1): 1-17.

VAN SLYKE, D.M. \& NEWMAN, H.K. (2006): "Venture Philanthropy and Social Entrepreneurship in Community Redevelopment", Case Study in Non Profit Management and Leadership, 16(3): 345368.

VARELA, R. (2001): Innovación empresarial: Arte y ciencia en la creación de empresas, $2^{\mathrm{a}}$ edición, Bogotá: Pearson Education.

WHITTINGTON, R. (2006): "Learning more from failures: practice and process", Organization Studies, 27(12): 1903-1906.

WOODSIDE, A.G. (2012): "Proposing a new logic for data analysis in marketing and consumer behavior: Case study research of large-N survey data for estimating algorithms that accurately profile $X$ (extremely high-use) consumers", Journal of Global Scholars of Marketing Science: Bridging Asia and the World, 22(4): 277-289.

WOODSIDE, A.G. \& ZHANG, M. (2013): "Cultural diversity and marketing transactions: Are market integration, large community size, and world religions necessary for fairness in ephemeral exchanges?", Psychology y Marketing, 30(3): 263-276.

ZAHRA, S.A., GEDAJLOVIC, E., NEUBAUM, D.O. \& SHULMAN, J.M. (2009): "A typology of social entrepreneurs: Motives, search processes and ethical challenges", Journal of Business Venturing, 24(5): 519-532. 
